\begin{tabular}{|c|c|}
\hline Title & Weight function determinations for shear cracks in reinforced concrete beams based on finite element method \\
\hline Author(s) & Deng, Pengru; Matsumoto, Takashi \\
\hline Citation & $\begin{array}{l}\text { Engineering Fracture Mechanics, 177, 61-78 } \\
\text { https://doi.org/10.1016/.engfracmech.2017.03.046 }\end{array}$ \\
\hline Issue Date & $2017-05-15$ \\
\hline Doc URL & http:/hdl .handle.net/2115/74005 \\
\hline Rights & 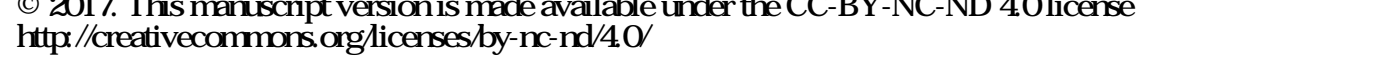 \\
\hline Rights(URL) & http://creativecommons.org/icenses/by-nc-nd/4.0/ \\
\hline Type & article (author version) \\
\hline File Information & Final manuscript.pdf \\
\hline
\end{tabular}

Instructions for use 


\title{
Weight Function Determinations for Shear Cracks in Reinforced Concrete Beams Based on Finite Element Method
}

\author{
Pengru Deng ${ }^{1}$ and Takashi Matsumoto ${ }^{2}$
}

${ }^{1}$ Graduate School of Engineering, Hokkaido University, Hokkaido 060-8628, Japan; Tel: +81-90-62135982; Email: dengpengru1989@gmail.com

${ }^{2}$ Faculty of Engineering, Hokkaido University, Hokkaido 060-8628, Japan; Tel: +81-11-706-6171; Fax: +81-11-706-6172; Email: takashim@eng.kokudai.ac.jp

\section{ABSTRACT:}

An efficient approach of evaluating the weight functions for the generally load applied faces of shear cracked RC beams with varying shear span/beam depth ratios is presented using the corresponding weight functions for the RC beams with two shear span/beam depth ratios. The shear cracked RC beam under applied load can be considered as an oblique edge crack elastic geometry under mixed load conditions if the nonlinear bridging force is evaluated according to a crack bridging model separately. With pure bend load conditions, both Mode I and II weight functions for the oblique edge crack geometries with three shear span/beam depth ratios for different crack length/beam depth ratios are evaluated through applying the Virtual Crack Extension technique with symmetric mesh around the crack-tip into finite element analysis, where all weigh function components exhibit orderly trends with respect to the changing shear span/beam depth. As a result, the weight functions for the shear crack RC beams with the other shear span/beam depth ratios can be calculated following the orderly trends. Since the crack-tip singular behaviors of fracture problems is observed only in the primary crack-face nodal 
weight functions, to facilitate application, the primary crack-face weight functions are formulated accurately as a function of crack length and distance away from the crack-tip employing the fitting and interpolation methods.

KEYWORDS: Shear crack; Reinforced concrete beam; Fracture mechanics; Weight function; Virtual Crack Extension technique;

\section{INTRODUCTION}

The oblique shear failure of reinforced concrete beams has long been known to be a brittle and catastrophic type of failure. Even though the behavior of reinforced concrete in shear has been studied for more than 100 years, the problem of how shear failures occur in RC beams still remains, by and large, unsolved as international codes, such as the American Concrete Institute (ACI) code (ACI, 1999) or the Eurocode 2 (Institution, 2004), are based on rather (semi-) empirical considerations.

The fracture mechanics provides a possible theoretical approach of analyzing the oblique shear crack of RC beams. Unfortunately, the concrete fracture process is complicated because a nonlinear strain softening, i.e., a negative slope in the stress-deformation diagram, is observed due to the localized crack process zone, which makes the Linear Elastic Fracture Mechanics (LEFM) seems inapplicable. However, the application of LEFM is not hindered at all by the seemingly nonlinear characteristics of the concrete cracking behavior following the idea of a fictitious crack mode proposed by (Hillerborg, Modéer, \& Petersson, 1976), where the nonlinear crack bridging effect is simplified as a decreasing function of the displacement discontinuity separately. In terms of the crack bridging model, several versatile models have been proposed based on the cohesive law (Carpinteri, Cornetti, Barpi, \& Valente, 2003) or through fitting experimental data (Stang \& Aarre, 1992). 
In LEFM, the weight function concept which was firstly proposed by (Bueckner, 1973; Rice, 1972) processes very strong advantages because the stress intensity factors for any arbitrary state of loading can be determined if the weight function of a given crack geometry is evaluated from a (perhaps simple) reference state of loading owing to load-independent characteristics of weight functions. Therefore, the use of weight function can obviate the repeated computer calculations of the stress intensity factors, which makes it suitable for some purposes, such as the structural life prediction where it is indispensable to analyze the cyclic load changes of a flawed structure. In addition, the weight function facilitated the formulation of the problem of a bridged crack in an elastic medium as an integral equation (Cox \& Marshall, 1991) illustrating the relation between crack opening displacement and crack bridging tractions. The integral equation was further exploited in determining the crack bridging forces (Nazmul \& Matsumoto, 2008a, 2008b).

Unfortunately, until now, the number of fracture problems with a close form analytical solution of weight function is very small. Because of that, different numerical methods suitable for the determination of weight functions have been presented, one of which is the Finite Element Method (FEM). The Virtual Crack Extension (VCE) technique, as suggested by (Hellen, 1975; Parks, 1974), provides an efficient finite element calculation of stress intensity factors and nodal weight functions. This technique has been employed in determining the 2-D Mode I weight functions in (George, 1984) and extended to 2-D Mixed Mode fracture problems in (George \& Chien-Tung, 1985) through the use of symmetric mesh in the vicinity of crack tip. Obviously, the oblique shear crack of RC beams can be simply considered as 2-D Mixed Mode fracture problems.

For the failure pattern of RC beams, one of the decisive factors is the size effect as reported in (Bazant \& Kazemi, 1991; Zhang \& Tan, 2007). (Muttoni \& Ruiz, 2008; Zararis \& Papadakis, 2001) concluded that the shear crack leading to failure occurs only in beam with a shear span to depth ration from 2.5 to 
8.0. Thus, weight functions for beam geometries with varying sizes are necessary, whereas the weight functions depend on the beam geometry. Therefore, this study is dedicate to presenting an approach of weight function determination for the shear cracked RC beams with varying shear span/beam depth ratio using only weight functions for beams with few shear span to beam depth ratios

\section{FORMULATION FOR MIXED FRACTURE MODE}

Exploiting the VCE technique in finite element method, the nodal weight functions for Mode I 2-D crack problems was represented in the displacement differentiation form according to the physical meaning of weight function, which is the normalized rate of change of displacements due to a unit change in the crack length for a reference state of loading, in (Rice, 1972). This computationally efficient finite element methodology for Mode I cracks was extended to mixed mode cracks, with combined tension and shear loading conditions, in (George \& Chien-Tung, 1985) through the use of symmetric mesh in the crack tip neighborhood. The symmetric mesh provides the decoupling characteristic of the stress, strain, displacement and traction field parameters into Mode I and Mode II components with respect to $x$ axis in the crack tip neighborhood as shown in Figure.1, as a result, the stress intensity factors and nodal weight functions are separated into Mode I and Mode II components. The decoupled nodal weight functions for Mode I and Mode II at i's nodal location $\left(x_{i}, y_{i}\right)$ with crack length $(a)$ and inclination angle $(\beta)$ can be represented in the displacement differentiation form as

$$
\begin{aligned}
& h_{I(I I) x}\left(x_{i}, y_{i}, a, \beta\right)=\frac{H}{2 K_{I(I I)}} \frac{\partial U_{I(I I) x}\left(x_{i}, y_{i}, a, \beta\right)}{\partial a} \\
& h_{I(I I) y}\left(x_{i}, y_{i}, a, \beta\right)=\frac{H}{2 K_{I(I I)}} \frac{\partial U_{I(I I) y}\left(x_{i}, y_{i}, a, \beta\right)}{\partial a}
\end{aligned}
$$

where

$$
h_{I(I I) x}=\text { weight function components along } x \text { axis for Mode I and Mode II }
$$


$h_{I(I I) y}=$ weight function components along $y$ axis for Mode I and Mode II

$K_{I(I I)}=$ stress intensity factors for Mode I and Mode II

$H=$ effective modulus which is $E$ for plane stress and $E /\left(1-v^{2}\right)$ for plane strain

$\mathrm{E}=$ Young's modulus

$v=$ Possion's ratio

$U_{I(I I) x}=$ displacement components along $x$ axis for Mode I and Mode II deformation

$U_{I(I I) y}=$ displacement components along $y$ axis for Mode I and Mode II deformation

The decoupled displacement components, $U_{I(I I) x}$ and $U_{I(I I) y}$, for Mode I and Mode II within the symmetric region in the crack tip neighborhood can be determined according to (Ishikawa, Kitagawa, \& Okamura, 1980)

Based on the definition of strain energy release rate, in VCE technique the strain energy release rate is the change in potential energy in a given loading system produced by the virtual crack extension. By applying the VCE technique with symmetric mesh in the crack tip neighborhood to the mixed mode fracture problems, the decoupled strain energy release rate $G_{I}$ for Mode I and $G_{I I}$ for Mode II can be obtained from the decomposed displacement components $\left\{U_{I}\right\}$ and $\left\{U_{I I}\right\}$, the changes in global stiffness $\Delta[K]$ and decomposed nodal force components $\Delta f_{I}$ and $\Delta f_{I I}$ as follows:

$$
\begin{aligned}
& G_{I}=-\frac{1}{2}\left\{U_{I}\right\}^{T} \frac{\partial[K]}{\partial a}\left\{U_{I}\right\}+\left\{U_{I}\right\}^{T} \frac{\partial\left\{f_{I}\right\}}{\partial a} \\
& G_{I I}=-\frac{1}{2}\left\{U_{I I}\right\}^{T} \frac{\partial[K]}{\partial a}\left\{U_{I I}\right\}+\left\{U_{I I}\right\}^{T} \frac{\partial\left\{f_{I I}\right\}}{\partial a}
\end{aligned}
$$

Then, $K_{I}$ and $K_{I I}$ can be obtained from the energy-based strain energy release rate according to the following relations

$$
G_{I} H=K_{I}^{2}
$$




$$
G_{I I} H=K_{I I}^{2}
$$

The decouple displacement derivatives, $\partial U_{I(I I) x}\left(x_{i}, y_{i}, a, \beta\right)$ and $\partial U_{I(I) y}\left(x_{i}, y_{i}, a, \beta\right)$ for the entire structure, can be obtained through the following process. To simplify the problem, the inclined angle $\beta$ is considered as constant for a given crack. For a given $\beta$, the decoupled Mode I and Mode II displacement components can be expressed functionally as

$$
\left\{U_{I(I I)}\right\}=\left\{U_{I(I I)}(x, y, a)\right\}
$$

Applying the chain rule of differentiation with respect to the crack length $(a)$ produces the following equation after rearrangement:

$$
\frac{\partial\left\{U_{I(I I)}\right\}}{\partial a}=\frac{d\left\{U_{I(I I)}\right\}}{d a}-\frac{\partial\left\{U_{I(I I)}\right\}}{\partial x} \cdot \frac{d x}{d a}-\frac{\partial\left\{U_{I(I I)}\right\}}{\partial y} \cdot \frac{d y}{d a}
$$

Since the stresses, strains and displacements of Mode I and Mode II are independent with each other and should satisfy the equilibrium equation and compatibility condition, we can obtain that

$$
[K]\left\{U_{I(I I)}\right\}-\left\{f_{I(I I)}\right\}=0
$$

$[K]$ is the matrix of global stiffness of original crack geometry.

Taking total differentiation of Eq.(6) with respect to crack length and after rearranging, we have

$$
\frac{d\left\{U_{I(I I)}\right\}}{d a}=[K]^{-1}\left[\frac{d\left\{f_{I(I I)}\right\}}{d a}-\frac{d[K]}{d a}\left\{U_{I(I I)}\right\}\right]
$$

As the changes of the elemental stiffness for the entire structure and the decoupled nodal forces occur only in a few elements around the crack tip as a result of VCE, $d[K] / d a$ and $d\left\{f_{I(I I)}\right\} / d a$ of Eq.(7) can be expressed as:

$$
\begin{aligned}
& \frac{d[K]}{d a}=\sum_{i=1}^{N_{c}} \frac{\left[k_{i}\right]_{a+\Delta a}-\left[k_{i}\right]_{a}}{\Delta a} \\
& \frac{d\left\{f_{I(I I)}\right\}}{d a}=\sum_{i=1}^{N_{f}} \frac{\left.\left.\left\{f_{I(I I)}\right)\right|_{a+\Delta a}-\left\{f_{I(I I)}\right)\right\}_{a}}{\Delta a}
\end{aligned}
$$


where

$$
\begin{aligned}
\Delta a & =\text { VCE in direction collinear with the oblique crack } \\
{\left[k_{i}\right] } & =\text { elemental stiffness matrix } \\
\left\{f_{I(I I)}\right\} & =\text { elemental matrix after VCE } \\
N_{c} & =\text { number of elements around the crack tip } \\
N_{f} & =\text { number of crack-face elements with nodal perturbation of } f_{I(I I)} \text { as a result of VCE }
\end{aligned}
$$

The last two terms of Eq.(5) serve as the correction factors of changing the total displacement derivatives to partial displacement derivatives for the oblique cracks, which are null for nodes without geometric changes as a result of VCE. For the VCE, which is collinear with an oblique crack, we have $d y / d a=0.0$

By substituting Eq.(5) into Eq.(1), the nodal weight functions for Mode I and Mode II, with crack length $(a)$ and inclination angle $(\beta)$ at $\left(x_{i}, y_{i}\right)$ locations, can be expressed as

$$
\begin{aligned}
& h_{I(I I) x}\left(x_{i}, y_{i}, a, \beta\right)=\frac{H}{2 K_{I(I I)}}\left\{\frac{d\left\{U_{I(I I) x}\right\}}{d a}-\frac{\partial\left\{U_{I(I I) x}\right\}}{\partial x} \frac{d x}{d a}\right\} \\
& h_{I(I I) y}\left(x_{i}, y_{i}, a, \beta\right)=\frac{H}{2 K_{I(I I)}}\left\{\frac{d\left\{U_{I(I I) y}\right\}}{d a}-\frac{\partial\left\{U_{I(I I) y}\right\}}{\partial x} \frac{d x}{d a}\right\}
\end{aligned}
$$

\section{MODELING IN FINITE ELEMENT ANALYSIS}

\subsection{Finite element model}

This study focuses on 2-D elastic plane problems and the finite element model is assumed under plane strain conditions where two material properties, Elastic modulus $E$ and Poisson's ratio $v$, need to be 
defined. As illustrated in the formulation section, the elements at the crack-tip neighborhood have a significant influence on the accuracy of the VCE technique

For the finite element modeling of fracture problems, firstly, the crack-tip singularity, $\sqrt{r}$ and $1 / \sqrt{r}$ displacement and stress variations, should be represented adequately in finite element method. Accordingly, the crack-tip vicinity is modeled with the degenerated quarter-point quadratic elements where the singularity is implicitly included. The degenerated triangular quarter-point element can be formed from standard 8-noded quadratic elements by defining duplicate node numbers for nodes $\mathrm{K}$, L and $\mathrm{O}$ and shift node $\mathrm{P}$ and $\mathrm{N}$ to the quarter-point as shown in Figure 2.

For the standard 8-noded plane isoparametric element, the geometry is mapped into the normalized square space shape $(\xi, \eta),(-1 \leq \xi \leq 1,-1 \leq \eta \leq 1)$ through the transformations as shown in Figure 3

$$
\begin{aligned}
& x^{c}=\sum_{i=1}^{8} N_{i}(\xi, \eta) x_{i}^{c} \\
& y^{c}=\sum_{i=1}^{8} N_{i}(\xi, \eta) y_{i}^{c} \\
& N_{i}(\xi, \eta)= {\left[\left(1+\xi \xi_{i}\right)\left(1+\eta \eta_{i}\right)-\left(1-\xi^{2}\right)\left(1+\eta \eta_{i}\right)-\left(1-\eta^{2}\right)\left(1+\xi \xi_{i}\right)\right] \xi_{i}^{2} \eta_{i}^{2} / 4 } \\
&+\left(1-\xi^{2}\right)\left(1+\eta \eta_{i}\right)\left(1-\xi_{i}^{2}\right) \eta_{i}^{2} / 2+\left(1-\eta^{2}\right)\left(1+\xi \xi_{i}\right)\left(1-\eta_{i}^{2}\right) \xi_{i}^{2} / 2
\end{aligned}
$$

where $\xi_{i}, \eta_{i}= \pm 1$ for the corner nodes and zero for the mid-side nodes, $x_{i}^{c}, y^{c}{ }_{i}$ are the nodal coordinates of the element. For the element shown in Figure 3(b) we have

$$
x_{1}^{c}=x_{7}^{c}=x_{8}^{c}=0 \quad x_{2}^{c}=x_{6}^{c}=\frac{h}{4} \quad x_{3}^{c}=x_{4}^{c}=x_{5}^{c}=h
$$

and

$$
y_{1}^{c}=y_{7}^{c}=y_{8}^{c}=y_{4}^{c}=0 \quad y_{2}^{c}=-y_{6}^{c}=-\frac{l}{4} \quad y_{3}^{c}=-y_{5}^{c}=-l
$$

Substituting Eq.(14) into Eq.(12) and after collecting terms 


$$
x^{c}=\frac{h}{4}(1+\xi)^{2}
$$

and

$$
y^{c}=\frac{l}{4} \eta(1+\xi)^{2}
$$

Therefore, the Jacobian of transformation $\left[J_{2}\right]$ between the normalized local coordinates $(\xi$, $\eta)$ and coordinates $\left(x^{c}, y^{c}\right)$ is given by

$$
\left[J_{2}\right]=\left[\begin{array}{ll}
\frac{\partial x^{c}}{\partial \xi} & \frac{\partial y^{c}}{\partial \xi} \\
\frac{\partial x^{c}}{\partial \eta} & \frac{\partial y^{c}}{\partial \eta}
\end{array}\right]=\left[\begin{array}{cc}
\frac{h}{2}(1+\xi) & \frac{l}{2} \eta(1+\xi) \\
0 & \frac{l}{4}(1+\xi)^{2}
\end{array}\right]
$$

and its determinant

$$
\operatorname{det}\left|J_{2}\right|=\frac{h l}{8}(1+\xi)^{3}
$$

Inverting

$$
\left[J_{2}\right]^{-1}=\left[\begin{array}{ll}
I_{11} & I_{12} \\
I_{21} & I_{22}
\end{array}\right]=\left[\begin{array}{cc}
\frac{2}{h(1+\xi)} & \frac{-4 \eta}{h(1+\xi)^{2}} \\
0 & \frac{4}{l(1+\xi)^{2}}
\end{array}\right]
$$

To facilitate calculation, the origin of the global coordinate system is located at the crack tip. As shown in Figure 1, the global coordinate system can be obtained through conducting a counterclockwise rotation by the angle, $\theta$. Thus, the formulae for coordinates $(x, y)$ can be expressed as

$$
\begin{aligned}
& x=x^{c} \cos \theta-y^{c} \sin \theta \\
& y=x^{c} \sin \theta+y^{c} \cos \theta
\end{aligned}
$$

Correspondingly, the Jacobian of transformation $\left[J_{1}\right]$ between the local coordinates $\left(x^{c}, y^{c}\right)$ and global coordinates $(x, y)$ is given by 
$\left[J_{1}\right]=\left[\begin{array}{ll}\frac{\partial x}{\partial x^{c}} & \frac{\partial y}{\partial x^{c}} \\ \frac{\partial x}{\partial y^{c}} & \frac{\partial y}{\partial y^{c}}\end{array}\right]=\left[\begin{array}{cc}\cos \theta & \sin \theta \\ -\sin \theta & \cos \theta\end{array}\right]$

Inverting

$$
\left[J_{1}\right]^{-1}=\left[\begin{array}{cc}
\cos \theta & -\sin \theta \\
\sin \theta & \cos \theta
\end{array}\right]
$$

Consequently, the Jacobian transformation of $[\mathrm{J}]$ between the normalized square shape local coordinates $(\xi, \eta)$ and global coordinates $(x, y)$ is given by

$$
[J]=\left[J_{2}\right] \cdot\left[J_{1}\right]
$$

Inverting

$$
[J]^{-1}=\left[J_{1}\right]^{-1} \cdot\left[J_{2}\right]^{-1}
$$

Taking the advantage of Jacobian matrix[J], the differentials of displacement with respect to the global coordinates $(x, y)$ for the isoparametric elements are given as

$$
\left\{\begin{array}{l}
\frac{\partial\left\{U_{I(I I)}\right\}}{\partial x} \\
\frac{\partial\left\{U_{I(I I)}\right\}}{\partial y}
\end{array}\right\}=[J]^{-1} \cdot\left\{\begin{array}{l}
\frac{\partial\left[N_{i} U_{I(I I) i}\right.}{\partial \xi} \\
\frac{\partial\left[N_{i} U_{I(I I) i}\right.}{\partial \eta}
\end{array}\right\}
$$

where $\left\{U_{I(I I) i}\right\}$ is the decoupled nodal displacement of Mode I or Mode II at $i$ 's nodal location. $N_{i}$ is the shape function at $i$ 's nodal location.

Similarly, the displacements $(u, v)$ within the element are interpolated by the same functions $N_{i}(\xi, \eta)$ of Eq.(13) from the nodal displacements $u_{i}, v_{i}$. Taking into account of the constraints, the nodal displacements of nodes 1, 7 and 8 are exactly the same, the differential of displacements in the local coordinates for any point at a distance $r$ from the crack tip on the radial line $R$ are given as

$$
\frac{\partial u}{\partial \xi}=a_{0}+a_{1}(1+\xi)
$$


$\frac{\partial u}{\partial \eta}=b_{1}(1+\xi)+b_{2}(1+\xi)^{2}$

$\frac{\partial v}{\partial \xi}=c_{0}+c_{1}(1+\xi)$

$\frac{\partial v}{\partial \eta}=d_{1}(1+\xi)+d_{2}(1+\xi)^{2}$

where $a_{0}, a_{1}, b_{1}, b_{2}, c_{0}, c_{1}, d_{1}, d_{2}$ are constants for any given set of nodal displacements along the radial line $R$. And it is obviously that

$r=\sqrt{\left(\left(x^{c}\right)^{2}+\left(y^{c}\right)^{2}\right)}$

Substituting Eq.(15) into Eq.(26) and after rearrangement, we get

$$
(1+\xi)=\sqrt{r} / \sqrt{\frac{l}{4}} \sqrt{\left[\left(\frac{h}{l}\right)^{2}+\eta^{2}\right]}
$$

And the derivatives $u$, and $v$ with respect to $x^{c}$ and $y^{c}$ reduce to

$$
\begin{aligned}
& \frac{\partial u}{\partial x^{c}}=\frac{A_{0}}{\sqrt{r}}+A_{1} \\
& \frac{\partial u}{\partial y^{c}}=\frac{B_{0}}{\sqrt{r}}+B_{1} \\
& \frac{\partial v}{\partial x^{c}}=\frac{C_{0}}{\sqrt{r}}+C_{1} \\
& \frac{\partial v}{\partial y^{c}}=\frac{D_{0}}{\sqrt{r}}+D_{1}
\end{aligned}
$$

where $A_{0}, A_{1}, B_{0}, B_{1}, C_{0}, C_{1}, D_{0}, D_{1}$ are also constants for any given set of nodal displacements and independent of $r$.

Therefore, the $\sqrt{r}$ displacement and correspondingly the $1 / \sqrt{r}$ stress characteristic of elasticity at the crack tip can be represented by the degenerated quarter-point quadratic elements, if the nodes 1, 7 and 8 at the crack tip are constrained to have the same displacement. 
Secondly, the result sensitivity for the element size is an eternal concern in finite element analysis especially for crack-tip elements in this study where the crack-tip elements determine the accuracy. The effect of the singular element size was previously studied by (Ingraffea \& Manu, 1980; Lynn \& Ingraffea, 1978). In both studies, triangular quarter-point elements surrounding the crack were employed with an outer mesh of quadrilateral elements. The results on stress intensity factors calculation showed that a local refinement of the mesh in the crack-tip zone improves the accuracy while an acceptable accuracy can be achieved even for relatively coarse mesh if the transition elements (regular eight-noded rectangular elements surrounding the triangular quarter-point elements) are employed (Banks-Sills \& Bortman, 1984; Chan, Tuba, \& Wilson, 1970). (Banks-Sills \& Bortman, 1984) pointed out that few percents error can be generally achieved for $0.1 \leq l_{s} / a \leq 0.3$, which satisfies almost all engineering purposes. The $1 \%$ level errors were achieved for $l_{s} / a=0.03$ in (Banks-Sills \& Bortman, 1984; Ingraffea \& Manu, 1980). Although from a practical point of view it does not seem desirable to use smaller elements, considering that error of stress intensity factors may be transformed to the nodal weight functions and then to the further fitting weigh functions, smaller element size with $l_{s} / a=0.04$ is employed in this study. Except for the crack-tip field, the remaining elastic body can be meshed with standard 8-noded quadratic elements.

\subsection{Result output}

According to the previous formulation on stress intensity factors and nodal weight functions for the combined mode I and mode II fracture problems, the global stiffness matrices and the nodal displacement vectors under a reference load should be output from finite element analysis to conduct

analysis of a combined mode I and mode II fracture problem based on the VCE technique. After conducting finite element analysis, we can get the nodal displacement vectors under the global 
coordinate system $(x, y)$. With these nodal displacement vectors, the Mode I and Mode II components for field displacements within the symmetric region in the crack tip neighborhood can be obtained following (Ishikawa, Kitagawa, \& Okamura, 1980), where a subtraction of corresponding nodal displacements between symmetrical nodes with respect to the $x$ axis is conducted. Since the corresponding nodal displacements of the symmetrical nodes are generally with very similar values, the

subtraction may eliminate all effective numbers if the digit length of the values is not enough. A subtraction is conducted between the global stiffness before and after the VCE to get the change of global stiffness due to the VCE as well. Due to the microscale VCE, the change of global stiffness matrices may be cut-off for matrix elements with insufficient digit length. Therefore, sufficient digit length for the element of nodal displacement vectors and global stiffness matrices should be ensured.

\section{RESULTS AND DISCUSSIONS}

This study is dedicated to providing some references for the fracture analysis of a major shear crack in an RC beam. In reality, the shear failure process involves the formation of multiple shear cracks before localization into a major crack. However, it is generally acceptable that a crack RC beam can be treated as an elastic body at the point where the static equilibrium reached because the sectional stiffness is mainly from rebars in the tension zone and concrete in the compression zone after cracking. Both rebars and compressed concrete should stay in the elastic state for an RC beam failure due to shear as the shear capacity is lower than the flexural capacity generally. Therefore, the derivation of weight functions based on elastic body assumption is applicable in determining the weight function for the major shear crack in an RC beam. The geometry and the finite element mesh of an oblique edge crack corresponding to the major shear crack are shown in Figure 4. Any constraint conditions can be employed for calculation and results discussion because as reported in (George \& Chien-Tung, 1985), the dependence 
of weight function on constrain conditions for a given crack geometry can be circumvented through combining all self-equilibrium forces, which include the applied surface tractions and the reaction forces included from the selected constraint conditions, with the nodal weight functions of different constraint conditions of the same crack geometry. The constraint conditions shown in Figure $\mathbf{5}$ are employed in all calculations in this study because the stress state for these constraint conditions is clarity and convenient for analyzing.

\subsection{Strain energy release rate}

Applying the VCE technique to the decoupling characteristics of the field parameters in the crack-tip neighborhood produces an efficient finite element evaluation for the mixed fracture mode for strain energy release rates. The accuracy assessment of the numerical strain energy release rates through the combination of the VCE technique and symmetrical mesh at the crack-tip vicinity is conducted through comparing with results from J-Integral method (Nishioka \& Atluri, 1983), which is a generally accepted approach of obtaining strain energy release rates, for oblique edge crack geometries with oblique angle $\beta=45^{\circ}, h_{2} / h_{1}=1.5, h / w=5$ and $0.1 \leq a / w \leq 0.8$ under pure bending load condition. The finite element mesh of a crack geometry and constraint conditions are shown in Figure 5. The J-Integral is originally formulated as a closed line-integral of strain energy density and work done by tractions around the crack tip and then extended to the finite element application based on a domain integral expression of $\mathrm{J}$ Integral derived in (Shih, Moran, \& Nakamura, 1986). The line integral method and the domain integral method were compared in (Li, Shih, \& Needleman, 1985), more extensive applicability and better accuracy have been achieved by the domain integral method. Therefore, the domain integral method is employed in obtaining J-Integral parameters (and hence strain energy release rates) for the elastic bodies in this study. 
Before conducting the VCE technique for strain energy release rates, the amount of collinear crack extension $\Delta a$ in Figure 1, which is one of the key operational parameters for the VCE technique, should be determined. (George, 1984) calculated strain energy release rates corresponding to a wide range of $10^{-14} \leq \Delta a / l_{s} \leq 10^{0}$ for Mode I problems and pointed out that there are two causes for the solution degradations. On one side, due mainly to the round-off error of the output global stiffness matrices and nodal displacement vectors, $\Delta a / l_{s}$ should not be small than $10^{-13}$, on the other side, when $\Delta a / l_{s}>10^{-3}$ the solution deterioration was observed due to the excessive shape distortion of the degenerated crack tip elements as a result of virtual crack extension. For practical purposes, $\Delta a / l_{s}$ ratio value of $10^{-5}$ was suggested for numerical computations on the Mode I problems. As the round-off error is one of the mainly causes for solution deterioration suggested from the study on Mode I problems, the solution stable range $\Delta a / l_{s}$ should be determined again for mixed mode problems even using the same precise level of stiffness and displacements. The reasons are: (1) The process of obtaining Mode I and Mode II field nodal displacements within the symmetric region in the crack tip neighborhood involves a subtraction of corresponding nodal displacements between symmetrical nodes with respect to the $x$ axis. This subtraction may cut off certain effective digits; (2) The Mode I and Mode II field nodal displacements may be in different orders of magnitude, as a result, the computation precisely sufficient for the calculation of Mode I strain energy release rates may be insufficient for the Mode II strain energy release rates. The normalized $G_{I}$ and $G_{I I}$ values corresponding to different $\Delta a / l_{s}$ are shown in Figure 6. It appears that normalized $G_{I}$ and $G_{I I}$ solutions approach the converged $G_{I}$ and $G_{I I}$ values as $\Delta a / l_{s}$ ratio decreases before showing accuracy degradation at $\Delta a / l_{s}$ ratio equaling $10^{-10}$ and $10^{-7}$, respectively, due to the round-off error mentioned in the upward sections. As the $\Delta a / l_{s}$ ratio increasing, both $G_{I}$ and $G_{I I}$ solution degradations are observed when $\Delta a / l_{s}>10^{-3}$. Therefore, for practical purposes, the midpoint, 
$\Delta a / l_{s}$ ratio value of $10^{-5}$ is used to generate the numerical results in this study, which is sufficient to ensure the solution stability.

With the $\Delta a / l_{s}$ ratio value of $10^{-5}$, the normalized strain energy release rate values evaluated from Virtual Crack Extension method for the oblique crack with different $a / w$ ratios are listed and compared with solutions from the domain integral method in Table 1. It is found that the maximum and average absolute discrepancy is $1.057 \%$ and $0.425 \%$ respectively between the solutions from these two methods. This confirms that the strain energy release rates of a combined mode I and mode II fracture problem can be evaluated accurately by applying VCE technique with symmetric mesh in the crack tip neighborhood.

\subsection{Weight function}

Even thought strain energy release rates and the corresponding stress intensity factors for mixed mode problems can be determined through applying the VCE technique into finite element analysis, it is still inconvenient to conduct calculations repeatedly for different load conditions. Therefore, a further step of this study is obtaining the weight function through applying the VCE technique into finite element analysis because once the weight functions for a particular cracked body is determined, the stress intensity factor for any loading system applied to the body can be calculated by a simple integration.

\subsubsection{Weight function for changing $h / w$ ratios}

For an RC beam, the failure mode is strongly dependency on the $h / w$ ratio. (Muttoni \& Ruiz, 2008) summarized the failure crack pattern of RC beams with $h / w$ varying from 1.5 to 8.0 under four-point bending load. It was found that the inclined shear crack leading to failure developed only in RC beams with $h / w$ ratios smaller than 8.0. For very large $h / w$, such as 8.0, only a series of almost parallel fracture 
cracks were observed on the failure RC beams. (Zararis \& Papadakis, 2001) reported that the critical inclined shear crack leading to collapse typically occurs only in slender beams, i.e., in beams with $h / w>2.5$. Therefore, the oblique crack geometries as shown in Figure 4(a) with oblique angle $\beta=45^{\circ}$ and $a / w=0.3,0.4,0.5,0.6$ and $h / w=2.5,5.0,7.5$ are employed for the detailed weight function calculation.

With respect to the $\left(x^{\prime}, y^{\prime}\right)$ coordinates, Figure 8 shows the weight function components along the lefthand face for the oblique edge crack geometries with $a / w=0.3,0.4,0.5,0.6$ and $h / w=2.5,5.0,7.5$ in relation to the distance away from the point $A$ in Figure 4. For a given group of $a / w$ and $h / w$ ratios, $h_{I x^{\prime}}$ and $h_{I I x^{\prime}}$ keep almost constant while $h_{I y^{\prime}}$ and $h_{I I y^{\prime}}$ decrease with an increase of the distance away from the point $A$. Specially, in all $h_{I x}$ and $h_{I I x^{\prime}}$, there is a consistent increase of absolute value with an increase of both $a / w$ ratio and $h / w$ ratio. For different $a / w$ ratios, the relation between the constant value of $h_{I x}$ and $h_{I I x^{\prime}}$ and $h / w$ ratios are shown in Figure 9(a) and Figure 9(b) respectively. It is found that for each a/w ratio $h_{I x}$ and $h_{I I x^{\prime}}$ increase linearly with the increasing of $h / w$, which means the $h_{I x}$ and $h_{I I x^{\prime}}$ for different $a / w$ and $h / w$ ratios can be obtained through linear interpolation of the presented results. In terms of $h_{I y^{\prime}}$ and $h_{I I y}$, they increase with respect to the increase of $a / w$ ratio, nevertheless stay nearly unchanged for a fixed $a / w$ ratio and changing $h / w$ ratio.

All the orderly trends represented in the curves of the weight function components can be interpreted theoretically as following. As shown in Figure 10, for a 2-D crack geometry subjected to any arbitrary combined Mode I and Mode I load condition, the linear elastic stress field around a crack tip can be expressed with as simple analytical form as

$$
\left\{\begin{array}{c}
\sigma_{x x} \\
\sigma_{y y} \\
\sigma_{x y}
\end{array}\right\}=\frac{K_{I}}{\sqrt{2 \pi r}}\left\{\begin{array}{c}
\cos (\theta / 2)[1-\sin (\theta / 2) \sin (3 \theta / 2)] \\
\cos (\theta / 2)[1+\sin (\theta / 2) \sin (3 \theta / 2)] \\
\sin (\theta / 2) \cos (\theta / 2) \cos (3 \theta / 2)
\end{array}\right\}+\frac{K_{I I}}{\sqrt{2 \pi r}}\left\{\begin{array}{c}
-\sin (\theta / 2)[2+\cos (\theta / 2) \cos (3 \theta / 2)] \\
\sin (\theta / 2) \cos (\theta / 2) \cos (3 \theta / 2) \\
\cos (\theta / 2)[1-\sin (\theta / 2) \sin (3 \theta / 2)]
\end{array}\right\}
$$

thus, for any point around the crack tip, $\theta$ and $r$ are constants. Then, 


$$
\left\{\begin{array}{l}
\sigma_{x x} \\
\sigma_{y y} \\
\sigma_{x y}
\end{array}\right\}=[C] \cdot\left\{\begin{array}{l}
K_{I} \\
K_{I I}
\end{array}\right\}
$$

where $[C]$ is a matrix of constants. According to the weight function concept, SIFs due to applied load is an integration of the applied load and the weight function at their points of application. Thus, the weight functions at a point on a crack geometry is equivalent to the SIFs if a unit concentrated load is applied on the point. Hence the $h_{I x}, h_{I I x^{\prime}}$ and $h_{I y^{\prime}}, h_{I I y^{\prime}}$ at a point on the left-hand face can be obtained if a unit load is applied at the point along the $x^{\prime}$ and $y^{\prime}$ axes, respectively. In the elastic mechanics scale, it is easily imaginable that the stresses of any point around the crack-tip stay the same if a unit load applied along the $x^{\prime}$ axis moving from point $A$ to $F$ in Figure 4 owing to the unchanged force lever from the crack-tip and vary linearly if the unit load applied along the $y^{\prime}$ axis. Similarly, following Eq. (30), the SIFs should experience the same trends manifested in the stresses. Therefore, $h_{I x}, h_{I I x^{\prime}}$ and $h_{I y^{\prime}}, h_{I I y^{\prime}}$ should be as shown in Figure 8.

Considering that loads are generally applied on the top-face of an RC beam, weight functions on the topface are presented as well in this study. Regarding the point $E$ which is closest point away from the crack-tip on the top-face as the origin and defining the direction from $F$ to $D$ as the positive direction, plots of the weight function components for the top-face of a series of typical crack geometries with $a / w=0.5$ and $h / w=2.5,5.0,7.5$ are shown in Figure 11. In $h_{I x}$ and $h_{I I x^{\prime}}$, a consistent decrease from maximum to almost zero is observed from point $F$ to $E$ due to the decreasing force lever, while the $h_{I y^{\prime}}$ and $h_{I I y^{\prime}}$ stay at a certain plateau in most of $F-E$ region and drop dramatically to almost zero just adjacent $E$. When the load applied location passes $E$, the force lever turns into zero and consequently both $h_{I x}, h_{I I x^{\prime}}$ and $h_{I y^{\prime}}, h_{I I y^{\prime}}$ remain almost zero in most of $E-D$ region. Due to the increasing content of local disturbing in the stresses around the crack-tip as the applied load approaching the crack-tip, a slight fluctuation is observed in all curves within a small region adjacent the origin point $E$. The characteristics shown in the 
weight function curves for the top-face can be interpreted similarly as for the left-hand face. Figure 12 shows the $h_{I x}, h_{I I x^{\prime}}$ and $h_{I y^{\prime}}, h_{I I y^{\prime}}$ weight function components along the top-face for the crack geometries with $a / w=0.3,0.4,0.5,0.6$ and $h / w=2.5,5.0,7.5$. For a given $a / w$ ratio, the characteristics exhibited in the series of typical geometries with $a / w=0.5$ and varying $h / w$ ratios are observed as well. Therefore, if a group of $h_{I x}, h_{I I x^{\prime}}$ and $h_{I y^{\prime}}, h_{I I y^{\prime}}$ weight function components for the top-face of a crack geometry with a span $(h)$ wider than the disturbed region is given, the weight function components for a crack geometry with a small span or a larger span can be obtained through cutting from or extending the given weight function components, respectively.

In general, an inclined shear crack in an RC beam is bridged by a series of forces acting on both crack faces owing to concrete aggregates or reinforcement. The evaluation of crack-face weight functions are of great significance. For the mixed-mode stress intensity factors, which are evaluated with the weight function concept with the crack-face loading, the explicit crack-face weight functions with respect to ( $x$, $y)$ coordinates $h_{I x}, h_{I I x}, h_{I y}$ and $h_{I I y}$ are more convenient to use than that of $\left(x^{\prime}, y^{\prime}\right)$ coordinates $h_{I x^{\prime}}, h_{I I x^{\prime}}, h_{I y^{\prime}}$ and $h_{I I y^{\prime}}$. The crack-face weight functions $h_{I x}, h_{I I x}, h_{I y}$ and $h_{I I y}$ along the lower and upper crack faces of the oblique edge crack geometries with $a / w=0.3,0.4,0.5,0.6$ and $h / w=2.5,5.0,7.5$ are shown respectively in Figure 13 and Figure 14, where $r_{s}$ is the distance away from the crack-tip. It is noted that the $1 / \sqrt{r}$ singular behaviors of the crack-face weight functions in crack-tip neighborhood are limited to the primary weight function components $\left(h_{I I x}\right.$ and $\left.h_{I y}\right)$ while the secondary crack-face weight functions $\left(h_{I x}\right.$ and $h_{I I y}$ ) are nonsingular in the crack-tip neighborhood. In addition, all series of weight functions for a fixed $a / w$ ratio stay almost the same with changing $h / w$ ratios, which means the repeated computation of crack-face weight functions for different $h / w$ ratios can be obviated for practical engineering purpose of determination of stress intensity factors with the weight function concept. 


\subsubsection{Weight function verification}

Considering the load independent characteristic of weight function, the nodal weight functions of the oblique edge crack geometries determined under the pure bending loads can be applied to the evaluation of stress intensity factors and the corresponding strain energy release rates for the mixed fracture mode under remote tension loads. The weight functions on the left-hand face of the oblique edge crack geometries with $h / w=5.0$ and $0.2 \leq a / w \leq 0.7$ are calculated using the pure bending load and shown in Figure 15. With the weight functions from pure bending loads, the strain energy release rates evaluated following weight function method and J-Integral method for the oblique edge crack elastic geometries under pure tension load conditions are listed in Table 2. The less than 1 percent discrepancies for all $a / w$ ratios further confirm the applicability and reliability of the weight functions determination for mixed mode fracture problems through applying VCE technique with symmetric mesh around crack-tip.

\subsubsection{Fitting and interpolation of weight functions}

Taking advantage of the well-known superposition principle, any load can be equivalent to a certain crack face load. As a result, the stress intensity factors due to the load may be determined with the crack-face weight functions which are obtainable through fitting the crack-face nodal weight functions with base functions. Generally, the polynomial bases are sufficient for the accurate fitting of the secondary crack-face nodal weight function components while the primary crack face weight function

components should be fitted with special bases because the $1 / \sqrt{r}$ singular behavior at the crack-tip neighborhood is observed in the primary crack-face nodal weight function components $h_{I y}$ and $h_{I I x}$ as illustrated in previous sections. It is obviously that the primary crack-face weight function components with a given crack length $(a)$ for the oblique edge crack geometry should be a function of the ratio $\left(r_{s} / a\right)$ between distance $\left(r_{s}\right)$ from the crack tip and the crack length $(a)$. Therefore, these primary components 
of a given oblique angle $\beta$ in $(x, y)$ coordinates can be fitting by the following equations where the superscripts $U$ and $L$ referring to the upper and lower crack faces respectively.

$$
\begin{aligned}
& h_{I y}^{U(L)}\left(r_{s} / a, a, \beta\right)=\sum_{n=1}^{N_{I}} A_{n}(a)\left(r_{s} / a\right)^{(n / 2-1)} \\
& h_{I I x}^{U(L)}\left(r_{s} / a, a, \beta\right)=\sum_{n=1}^{N_{I I}} B_{n}(a)\left(r_{s} / a\right)^{(n / 2-1)}
\end{aligned}
$$

where

$$
A_{n}(a)=\text { coefficients that are a function of crack length for a given oblique angle } \beta \text {. }
$$

$N_{I(I I)}=$ maximum number of terms required to achieve accurate fitting of the nodal weight functions. $N_{I}=6$ and $N_{I I}=6$ are found to provide accurate fitting results of for oblique edge crack geometry. Both the primary nodal weight functions and the fitting weight function curves along the lower and upper crack faces for the oblique edge crack geometries with $\beta=45^{\circ}$ and $0.1 \leq a / w \leq 0.8$ are shown in Figure 16. For the crack-tip node, the nodal weight function is unobtainable because the determinant of Jacobian matrix $[J]$ as illustrated in Eq.(23) approaches to infinite for this node. The coefficients, $A_{n}(a)$ and $B_{n}(a)$ corresponding to different crack lengths are listed in Table 3.

In terms of the application of weight functions, in some cases, such as the calculation of crack opening displacements based on the integral equation in (Cox \& Marshall, 1991), the weight function should be formulated a function of both crack length (a) and distance ratio away from the crack tip $\left(r_{s} / a\right)$. Thus, in this study, the coefficients, $A_{n}(a)$ and $B_{n}(a)$, are expressed as a function of crack length (a) for a given oblique angle through interpolating the corresponding coefficients for different crack length shown in Table 3. The applicability and reliability of the interpolation is confirmed through the less than $1 \%$ discrepancy between the weight functions obtained from applying the interpolated weight functions and the direct VCE technique for $a / w=0.35,0.45,0.55$ as shown in Figure 17. 


\section{CONCLUSIONS}

The fracture mechanics provides a possible theoretical approach of analyzing a shear crack in an RC beam. Following the idea of fictitious crack model, the RC beam with a shear crack can be regarded as an oblique edge crack elastic geometry if the nonlinear bridging force is considered according to a bridging model separately, and correspondingly the seemingly inapplicable LEFM can be employed.

Obviously, the oblique edge crack geometry is generally under mixed mode load conditions for which closed form analytical solutions are unavailable. Thus, an efficient finite element method, where the VCE technique is coupled with symmetric mesh in the crack-tip neighborhood, is used in evaluating both strain energy release rates and weight function for the oblique edge crack geometry. Specific findings and conclusions are summarized as follows:

(1) The stable ranges of the microscale crack extension ratio $\Delta a / l_{s}$ for Mode I and Mode II components are different even using the same precise stiffness matrices and nodal displacement vectors. The stable range should be determined beforehand for a mixed mode fracture problem.

(2) Accuracy assessment of strain energy release rate evaluation based on the VCE technique method is conducted through comparing the results from the VCE technique and the generally accepted J-Integral method for the oblique crack geometries with $h / w=5.0$ and changing $a / w$ ratios, where less than $1 \%$ discrepancies are achieved in almost all cases.

(3) The weight functions on all generally load applied faces for an RC beam, such as the left-hand face, top-face, upper and lower crack faces, are evaluated for the crack geometries with $h / w=2.5,5.0,7.5$ and $a / w=0.3,0.4,0.5,0.6$. In LEFM, for an elastic cracked geometry under any arbitrary load conditions, the stresses of any points around the crack-tip can be related to the Mode I and Mode II stress intensity factor with a constant matrix. In addition, the stresses vary linearly for a unit load moving along the boundaries of the cracked geometry except for the crack faces. Thus, the weight functions should vary 
linearly along the boundaries as is observed in all weight functions along the left-hand face and top face for the RC beam. Therefore, the weight functions along all boundaries except for the crack faces of an RC beam with any shear span/beam depth ratio can be evaluated using the corresponding weight functions for two RC beams with different shear span/beam depth ratios.

(4) In terms of the crack-face weight functions, the weight functions along both the upper and lower faces for an RC beam can be utilized in RC beams with different shear span/beam depth ratios directly because the influence of the changing shear span/beam depth ratios on crack-face weight functions is negligible for an engineering purpose. Conclusively, the weight function components on the generally load applied faces of the oblique edge crack geometries and the corresponding the shear cracked RC beams with varying shear span/beam depth ratios $(h / w)$ can be evaluated with the corresponding weight function components from only two shear span/beam depth $(h / w)$ ratios.

(5) For the same crack geometries, the weight functions obtained from pure bending load conditions are employed in evaluating the stress intensity factors and corresponding strain energy release rates due to pure tension loads according to the weight function concept. The less than $1 \%$ discrepancies between the normalized stress energy release rates from the weight function concept and J-Integral method confirms the reliability and applicability of evaluating weight functions using the VCE technique.

(6) To facilitate the application of weight functions, the primary crack-face weight functions are formulated successfully as a function of crack length $a$ and distance away from the crack-tip $r_{s}$ through fitting and interpolating the primary crack-face nodal weight functions. The fitted and interpolated weight functions are employed in calculating the crack-face weight functions for $a / w=0.35,0.45,0.55$ and then compared with the corresponding nodal weight functions obtained directly from VCE technique. The less than $1 \%$ discrepancy between the primary crack-face weight functions from these two approaches confirms the applicability and reliability of the fitting and interpolating process. 


\section{REFERENCES}

ACI, B. (1999). 318-Building Code Requirements for Reinforced Concrete and Commentary. American Concrete Institute International.

Banks-Sills, L., \& Bortman, Y. (1984). Reappraisal of the quarter-point quadrilateral element in linear elastic fracture mechanics. International journal of fracture, 25(3), 169-180.

Bazant, Z. P., \& Kazemi, M. T. (1991). Size effect on diagonal shear failure of beams without stirrups. ACI Structural Journal, 88(3), 268-276.

Bueckner, H. F. (1973). Field singularities and related integral representations. Methods of analysis and solutions of crack problems (pp. 239-314): Springer Netherlands.

Carpinteri, A., Cornetti, P., Barpi, F., \& Valente, S. (2003). Cohesive crack model description of ductile to brittle size-scale transition: dimensional analysis vs. renormalization group theory. Engineering Fracture Mechanics, 70(14), 1809-1839.

Chan, S., Tuba, I., \& Wilson, W. (1970). On the finite element method in linear fracture mechanics. Engineering Fracture Mechanics, 2(1), 1-17.

Cox, B., \& Marshall, D. (1991). The determination of crack bridging forces. International journal of fracture, 49(3), 159-176.

George, T. S. (1984). Stiffness derivative finite element technique to determine nodal weight functions with singularity elements. Engineering Fracture Mechanics, 19(4), 685-699.

George, T. S., \& Chien-Tung, Y. (1985). Weight function calculations for mixedmode fracture problems with the virtual crack extension technique. Engineering Fracture Mechanics, 21(6), 1119-1149.

Hellen, T. (1975). On the method of virtual crack extensions. International journal for numerical methods in engineering, 9(1), 187-207. 
Hillerborg, A., Modéer, M., \& Petersson, P.-E. (1976). Analysis of crack formation and crack growth in concrete by means of fracture mechanics and finite elements. Cement and concrete research, 6(6), 773-781.

Ingraffea, A. R., \& Manu, C. (1980). Stress - intensity factor computation in three dimensions with quarter - point elements. International journal for numerical methods in engineering, 15(10), 1427-1445.

Institution, B. S. (2004). Eurocode 2: Design of Concrete Structures: Part 1-1: General Rules and Rules for Buildings: British Standards Institution.

Ishikawa, H., Kitagawa, H., \& Okamura, H. (1980). J integral of a mixed mode crack and its application. Mechanical behaviour of materials, 447-455.

Li, F. Z., Shih, C. F., \& Needleman, A. (1985). A comparison of methods for calculating energy release rates. Engineering Fracture Mechanics, 21(2), 405-421.

Lynn, P. P., \& Ingraffea, A. R. (1978). Transition elements to be used with quarter - point crack - tip elements. International journal for numerical methods in engineering, 12(6), 1031-1036.

Muttoni, A., \& Ruiz, M. F. (2008). Shear strength of members without transverse reinforcement as function of critical shear crack width. ACI Structural Journal, 105(2).

Nazmul, I., \& Matsumoto, T. (2008a). High resolution COD image analysis for health monitoring of reinforced concrete structures through inverse analysis. International journal of solids and structures, 45(1), 159-174.

Nazmul, I., \& Matsumoto, T. (2008b). Regularization of inverse problems in reinforced concrete fracture. Journal of Engineering Mechanics, 134(10), 811-819. 
Nishioka, T., \& Atluri, S. (1983). Path-independent integrals, energy release rates, and general solutions of near-tip fields in mixed-mode dynamic fracture mechanics. Engineering Fracture Mechanics, 18(1), $1-22$.

Parks, D. M. (1974). A stiffness derivative finite element technique for determination of crack tip stress intensity factors. International journal of fracture, 10(4), 487-502.

Rice, J. R. (1972). Some remarks on elastic crack-tip stress fields. International journal of solids and structures, 8(6), 751-758.

Shih, C., Moran, B., \& Nakamura, T. (1986). Energy release rate along a three-dimensional crack front in a thermally stressed body. International journal of fracture, 30(2), 79-102.

Stang, H., \& Aarre, T. (1992). Evaluation of crack width in FRC with conventional reinforcement. Cement and Concrete Composites, 14(2), 143-154.

Zararis, P. D., \& Papadakis, G. C. (2001). Diagonal shear failure and size effect in RC beams without web reinforcement. Journal of structural engineering, 127(7), 733-742.

Zhang, N., \& Tan, K.-H. (2007). Size effect in RC deep beams: Experimental investigation and STM verification. Engineering Structures, 29(12), 3241-3254. 


\section{FIGURE LEGENDS}

Figure 1 Symmetric mesh in crack-tip neighborhood with respect to the global $x$ axis

Figure 2 Element geometry

Figure 3 2-D triangular quarter point elements and the parent element

Figure 4 Geometry and Finite Element Model for an oblique edge crack

Figure 5 Finite element model under different loads

Figure 6 Effect of incremental crack size of virtual crack extension on solution stability

Figure 8 Nodal weight functions left-hand face

Figure 9 Nodal weight functions left-hand face for different $a / w$ ratio

Figure 10 Elastic stress field at crack-tip

Figure 11 Typical nodal weight functions on top-face with $a / w=0.5$

Figure 12 Nodal weight functions on top-face

Figure 13 Nodal weight functions on upper crack-face

Figure 14 Nodal weight functions on lower crack-face

Figure 15 Nodal weight functions on top-face

Figure 16 Primary nodal weight functions and fitting primary weight functions

Figure 17 Primary weight functions from interpolated results and the VCE technique 


\section{FIGURES}

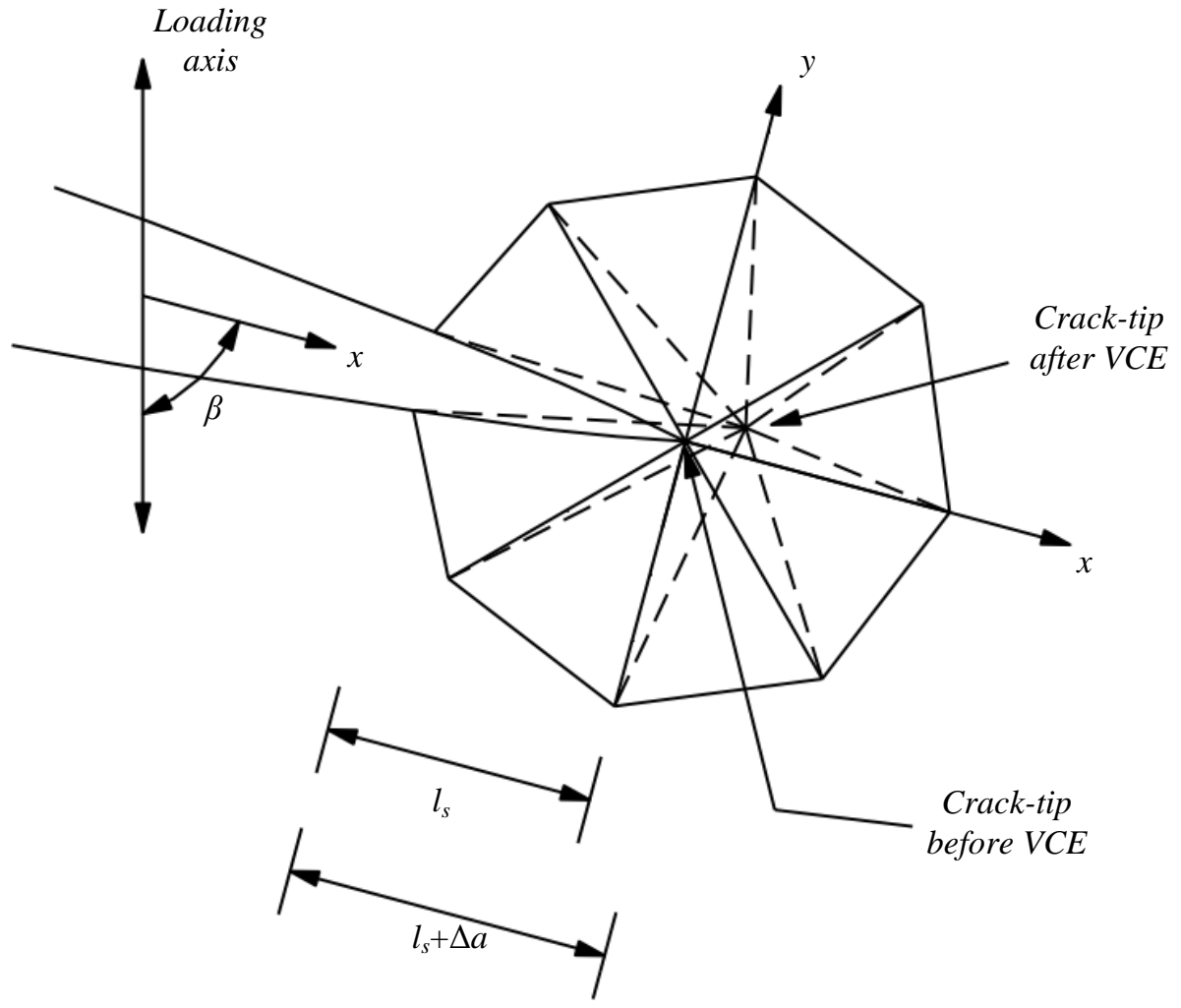

Figure 1 Symmetric mesh in crack-tip neighborhood with respect to the global $x$ axis 

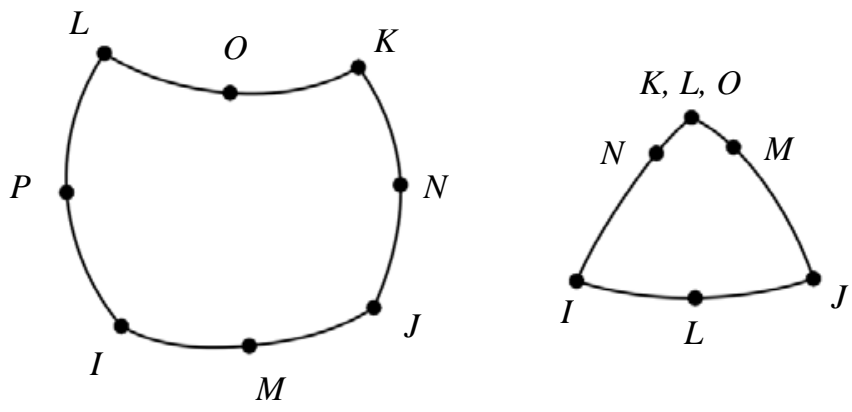

Figure 2 Element geometry 

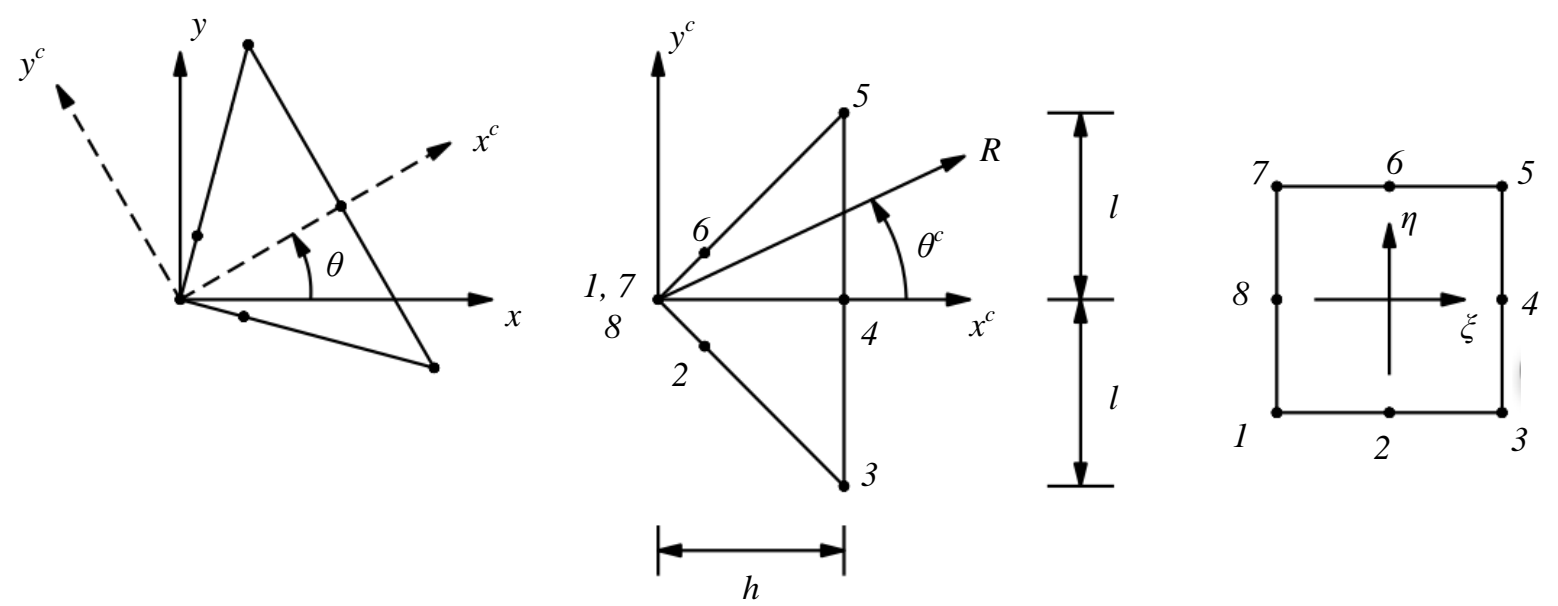

(a) In $x-y$ coordinate

(b) In $x^{c}-y^{c}$ coordinate

(c) In $\xi-\eta$ coordinate

Figure 3 2-D triangular quarter point elements and the parent element 

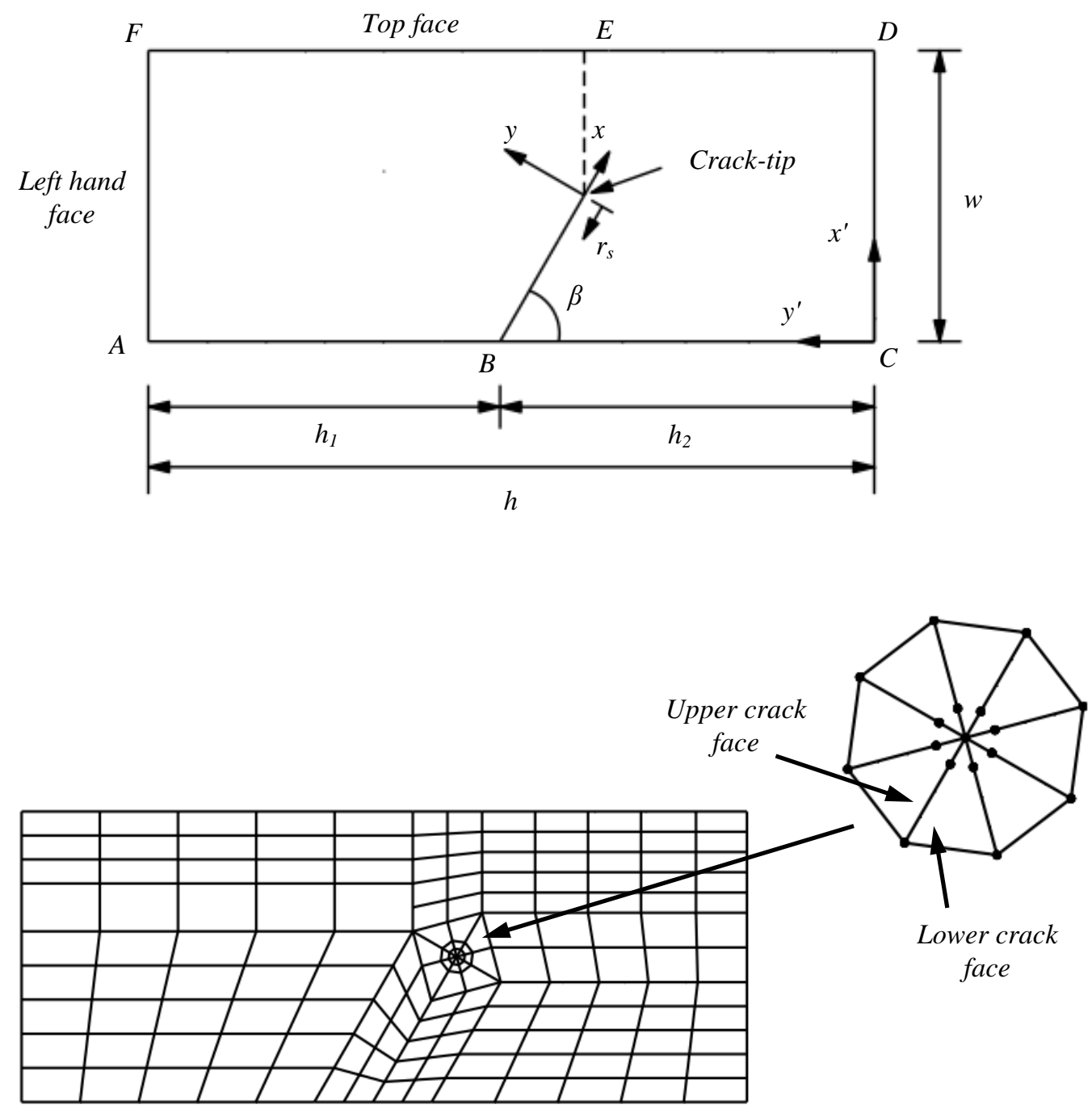

Figure 4 Geometry and Finite Element Model for an oblique edge crack 

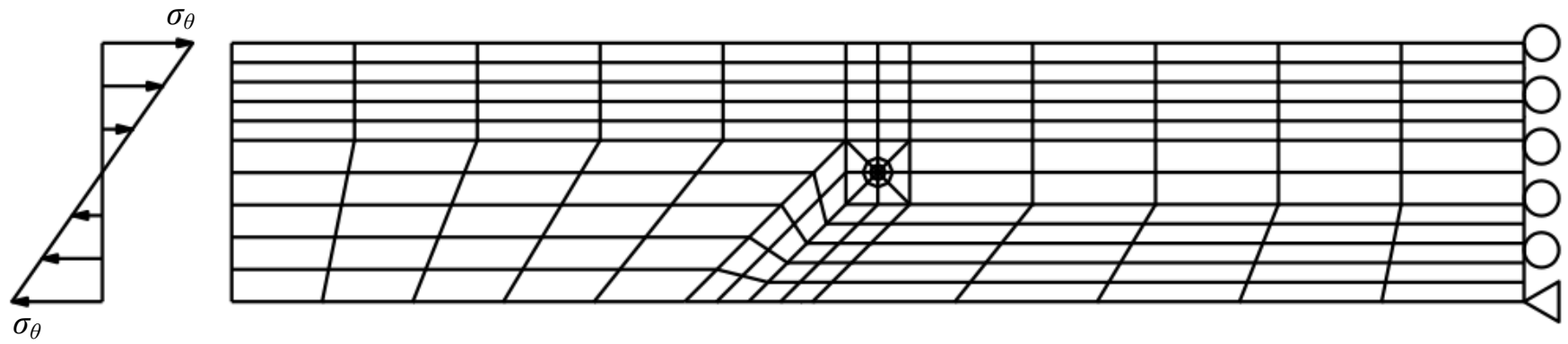

(a) Pure bending load

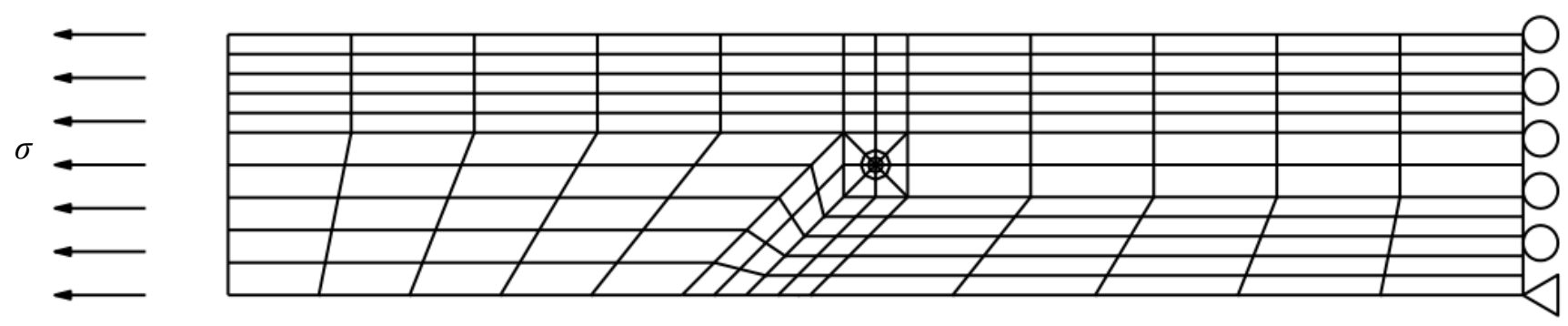

(b) Pure tension load

Figure 5 Finite element model under different loads 


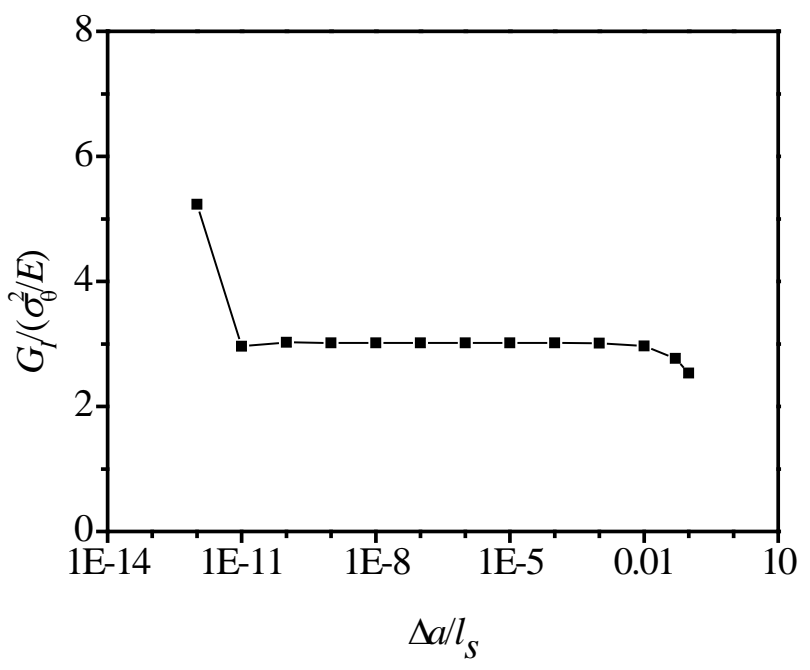

(a)

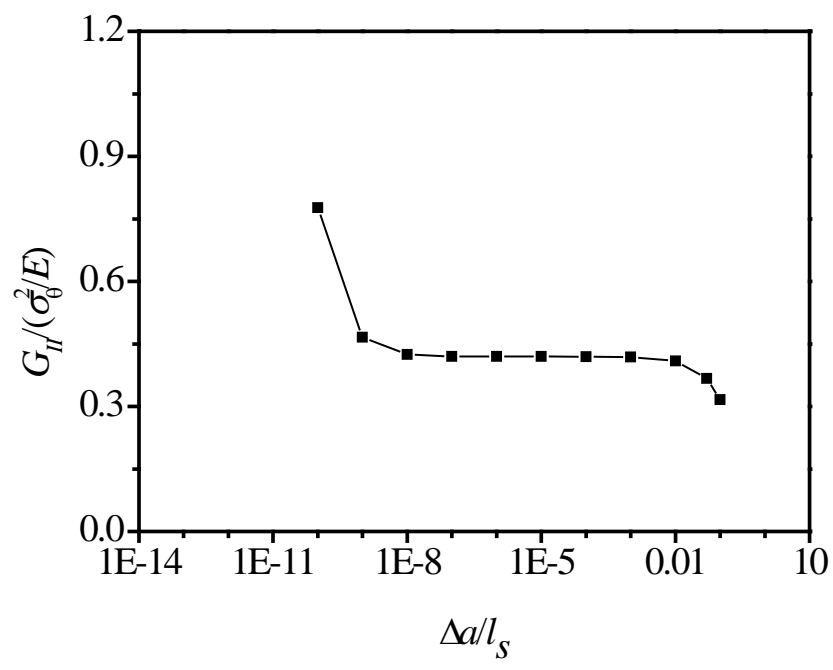

(b)

Figure 6 Effect of incremental crack size of virtual crack extension on solution stability 


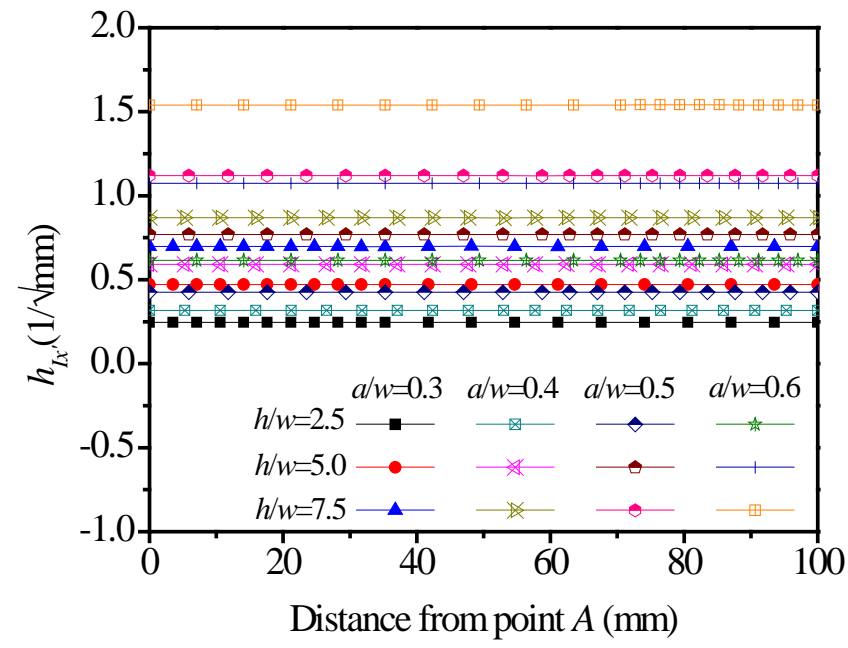

(a)

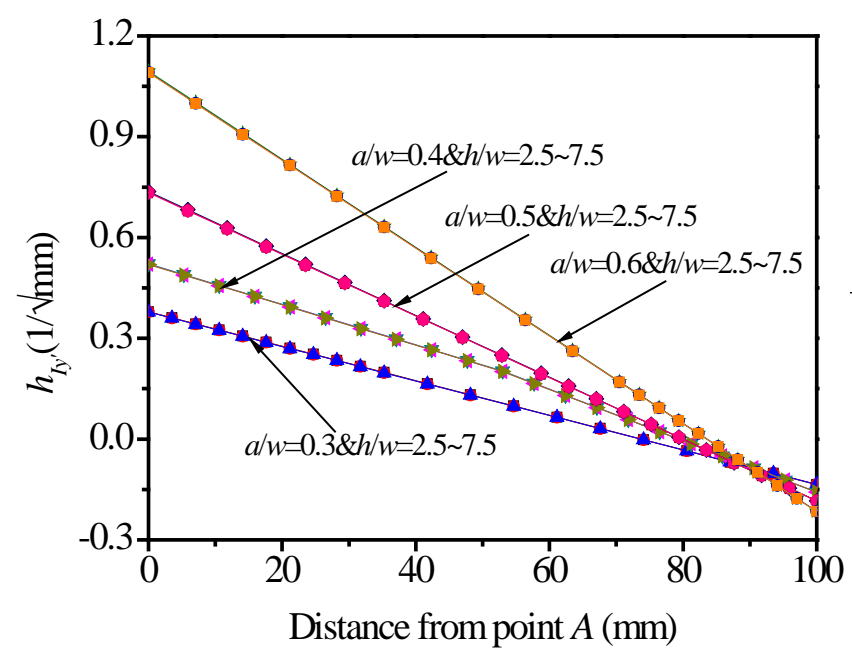

(c)

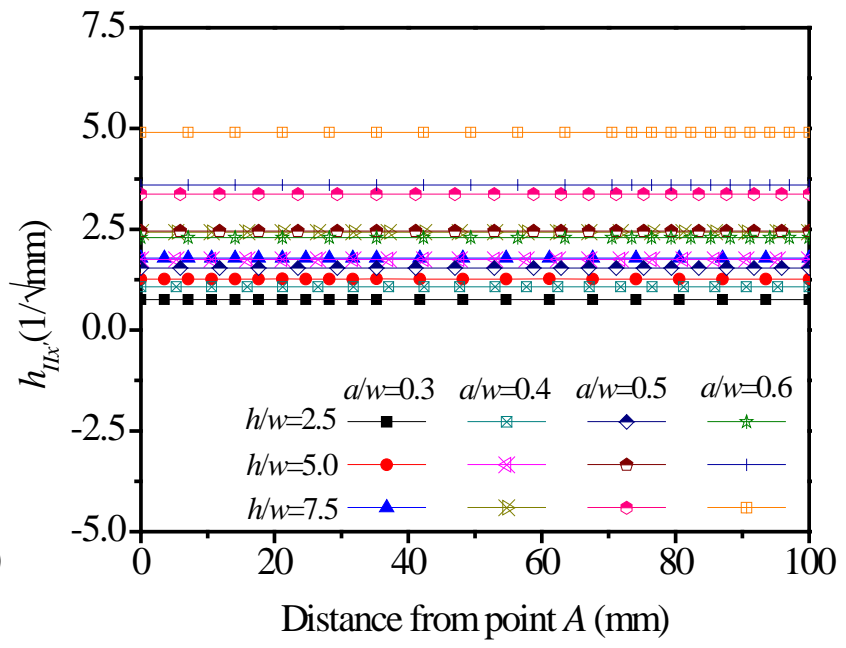

(b)

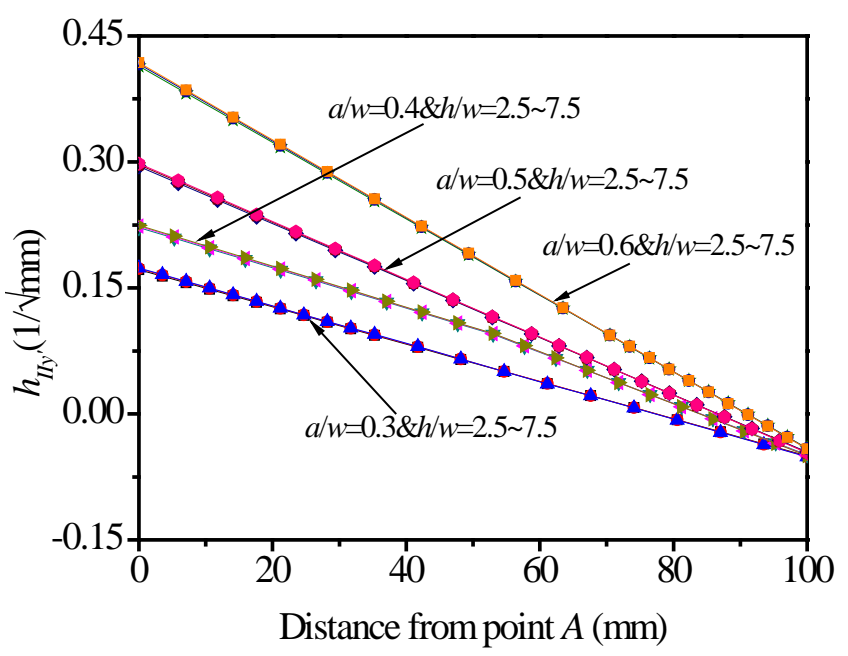

(d)

Figure 8 Nodal weight functions left-hand face 


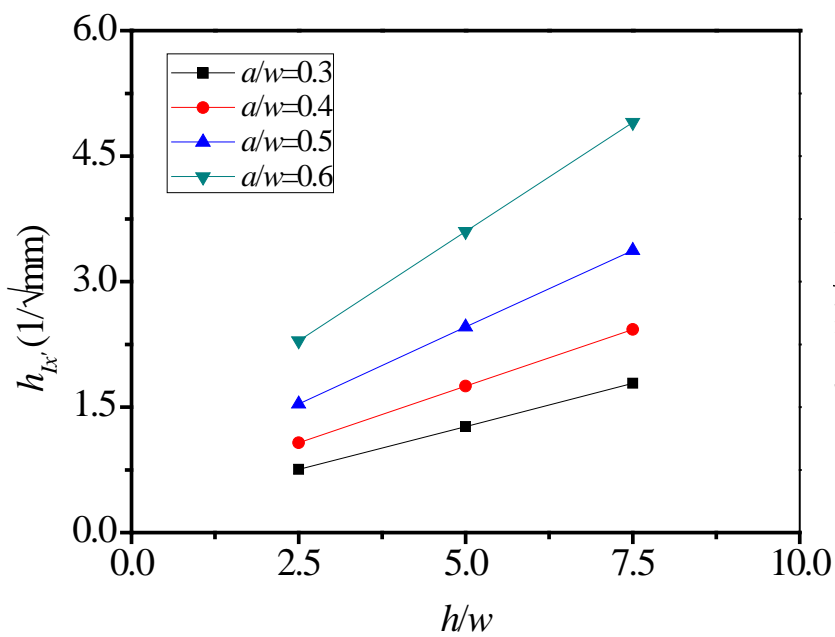

(a)

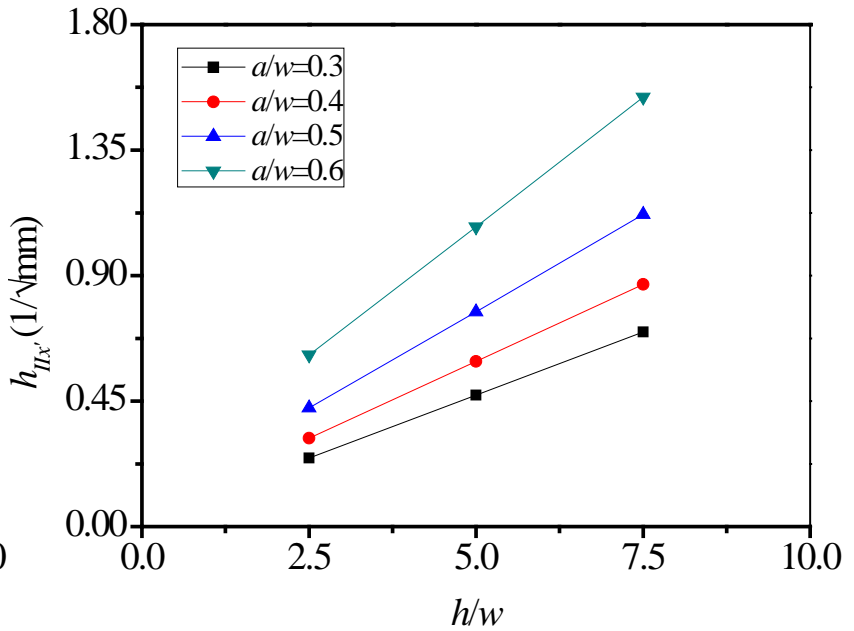

(b)

Figure 9 Nodal weight functions left-hand face for different $a / w$ ratio 


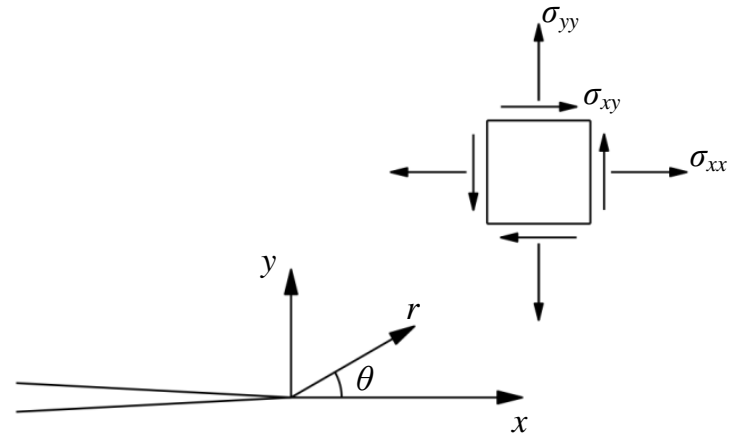

Figure 10 Elastic stress field at crack-tip 


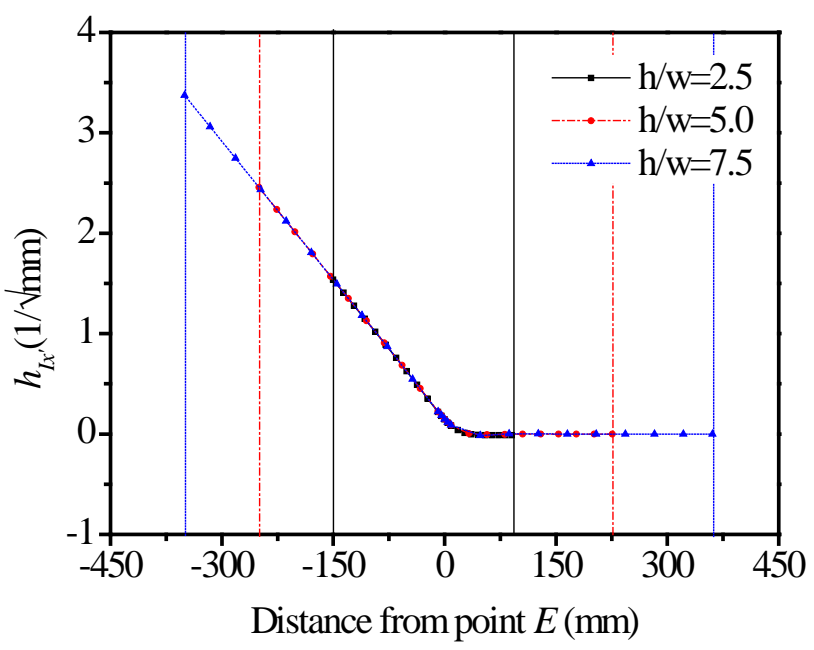

(a)

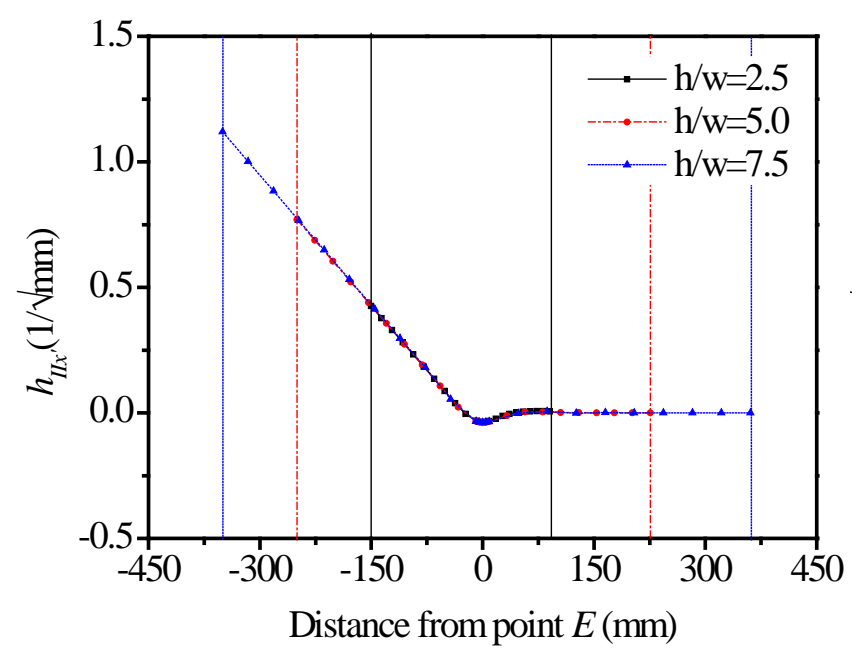

(c)

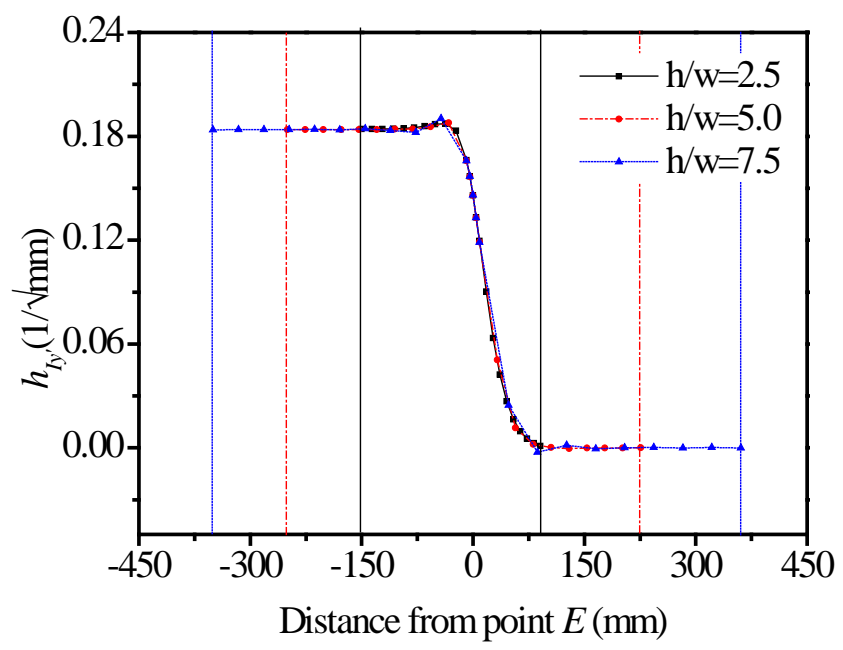

(b)

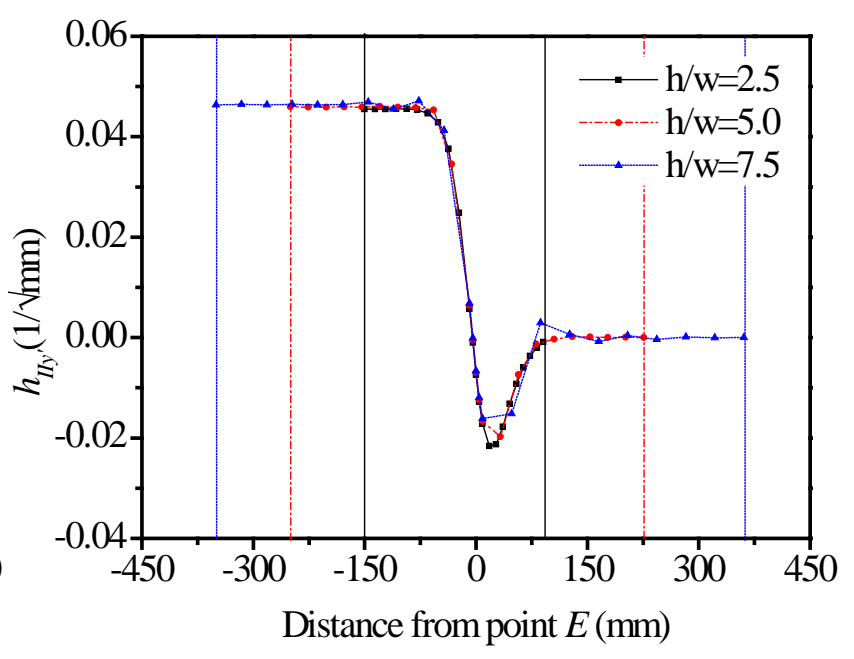

(d)

Figure 11 Typical nodal weight functions on top-face with $a / w=0.5$ 


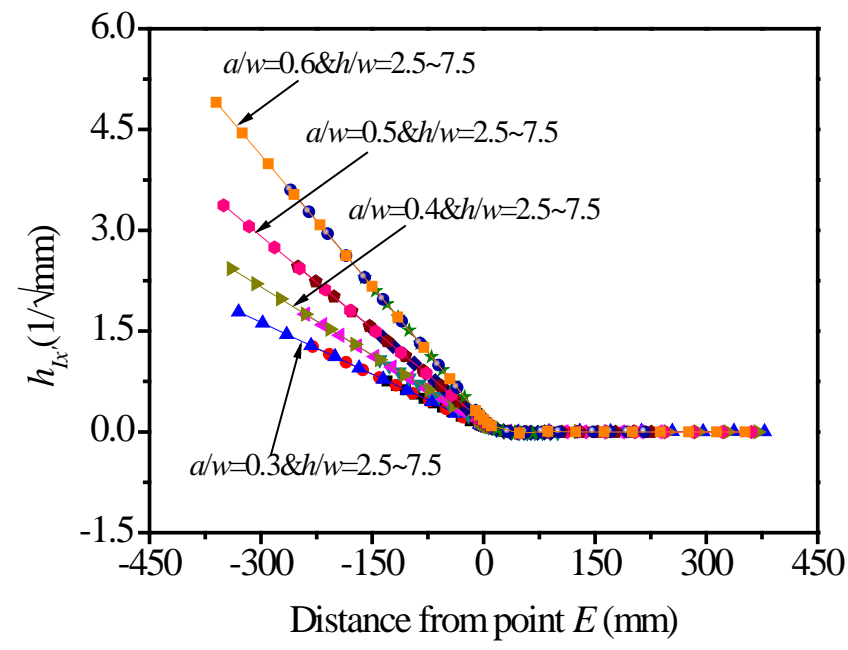

(a)



(c)

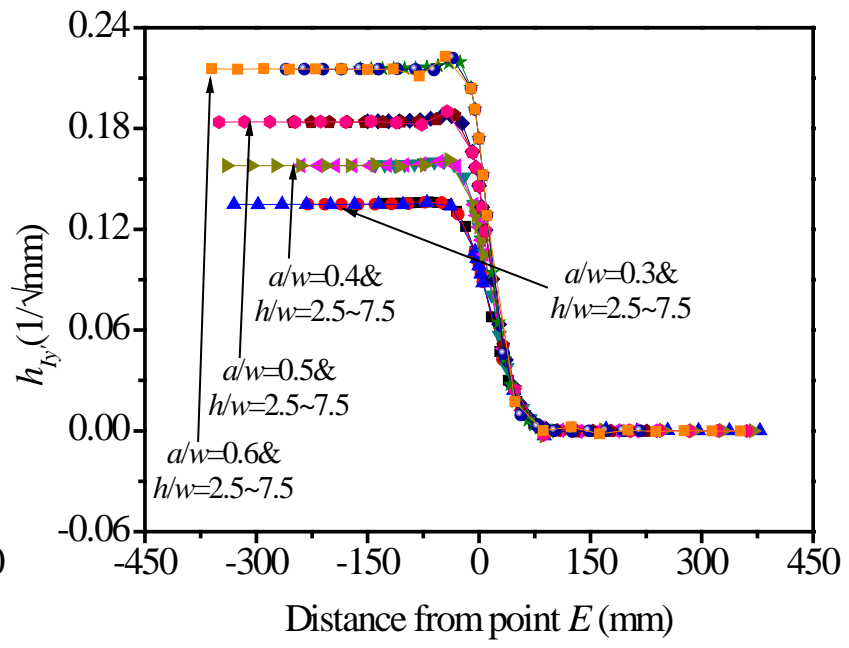

(b)



(d)

Figure 12 Nodal weight functions on top-face 


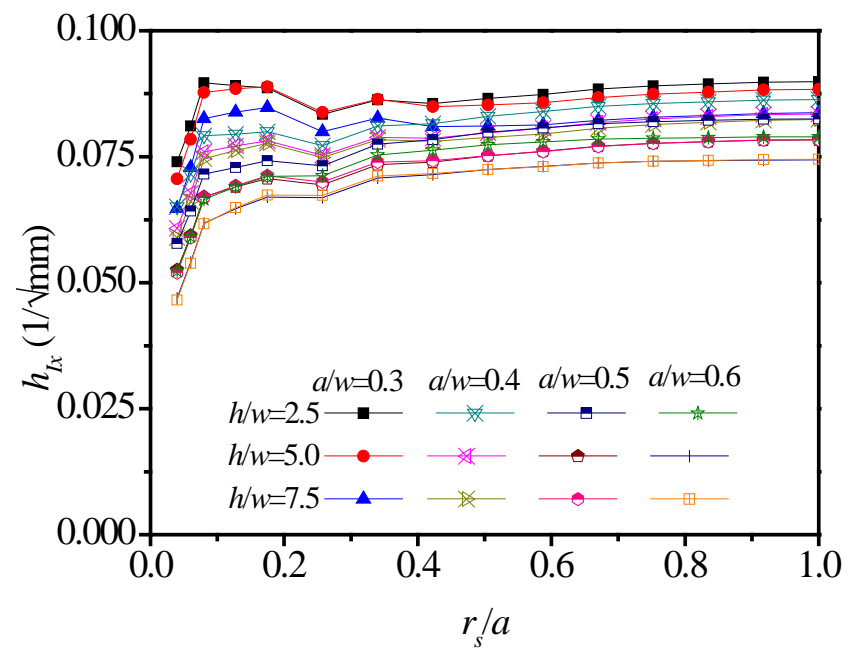

(a)

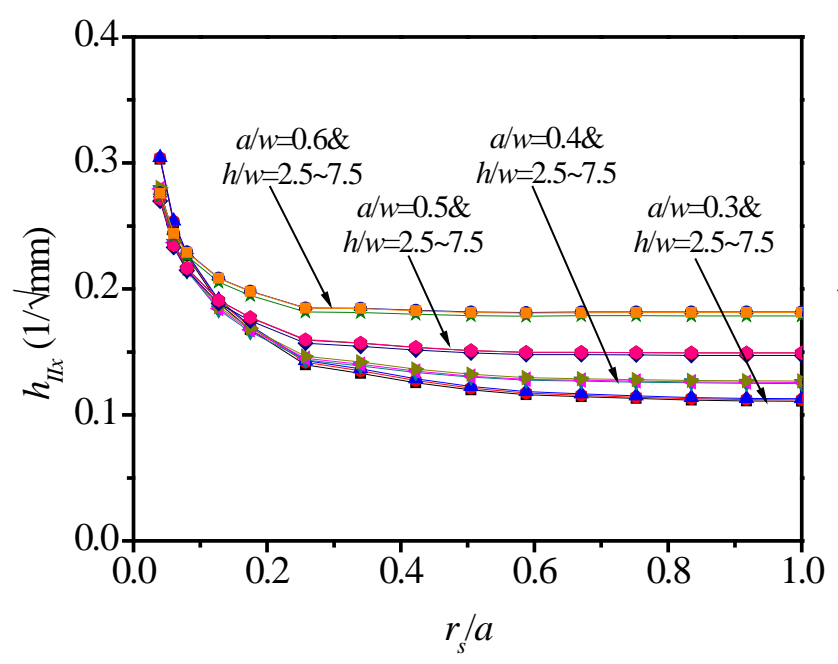

(c)

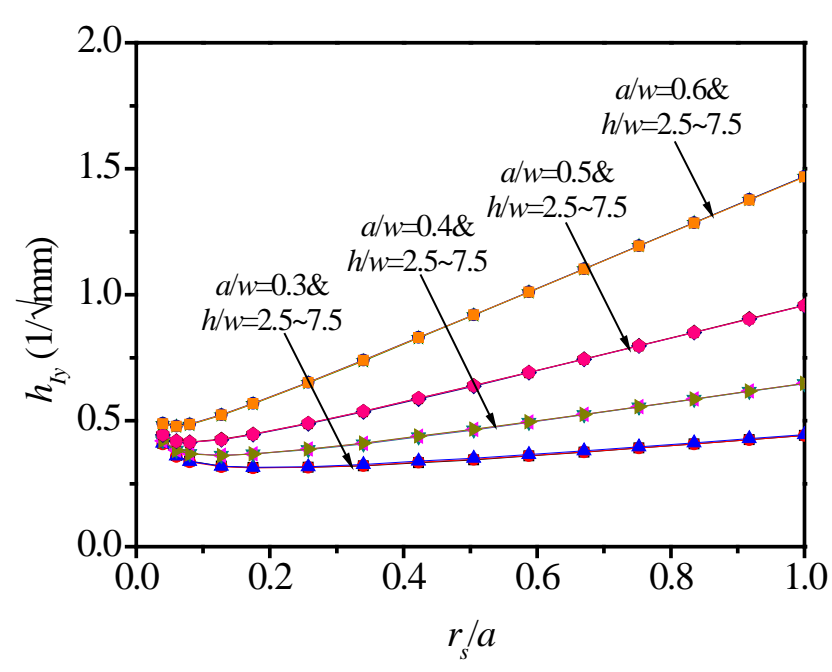

(b)

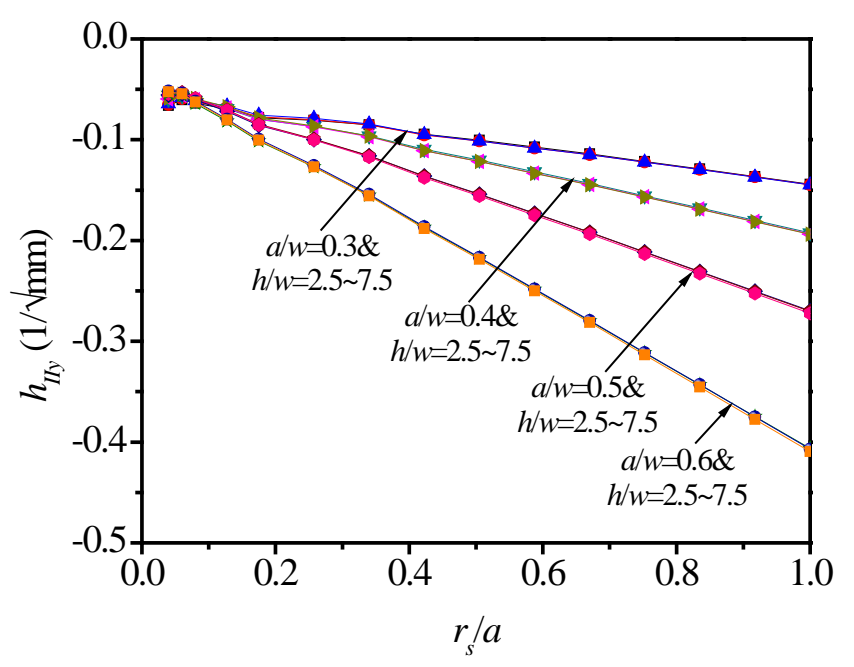

(d)

Figure 13 Nodal weight functions on upper crack-face 


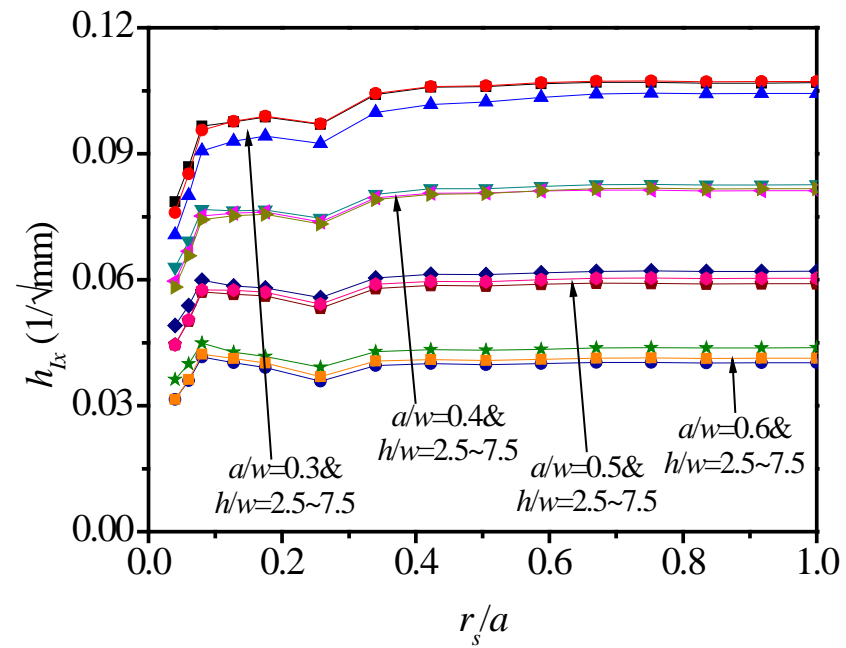

(a)

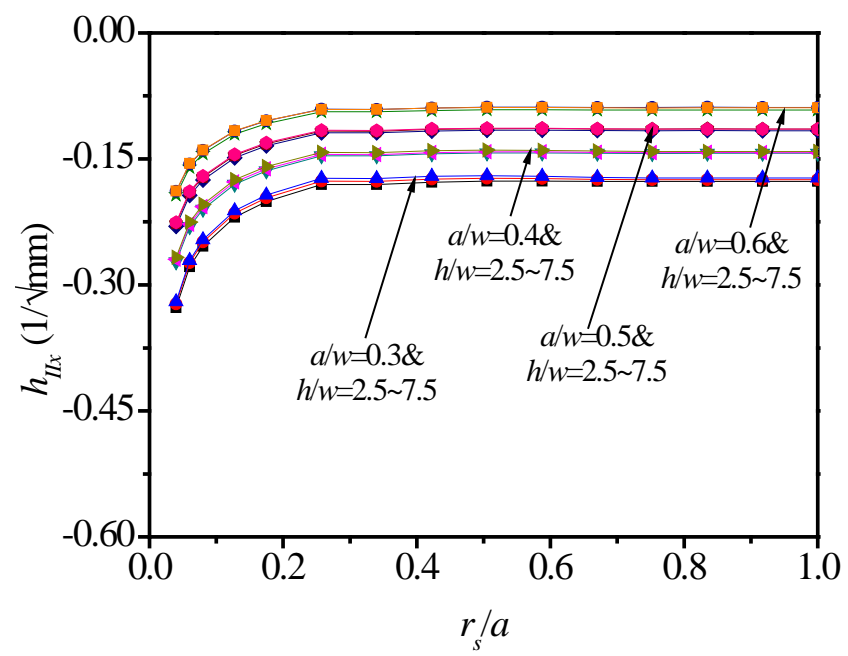

(c)

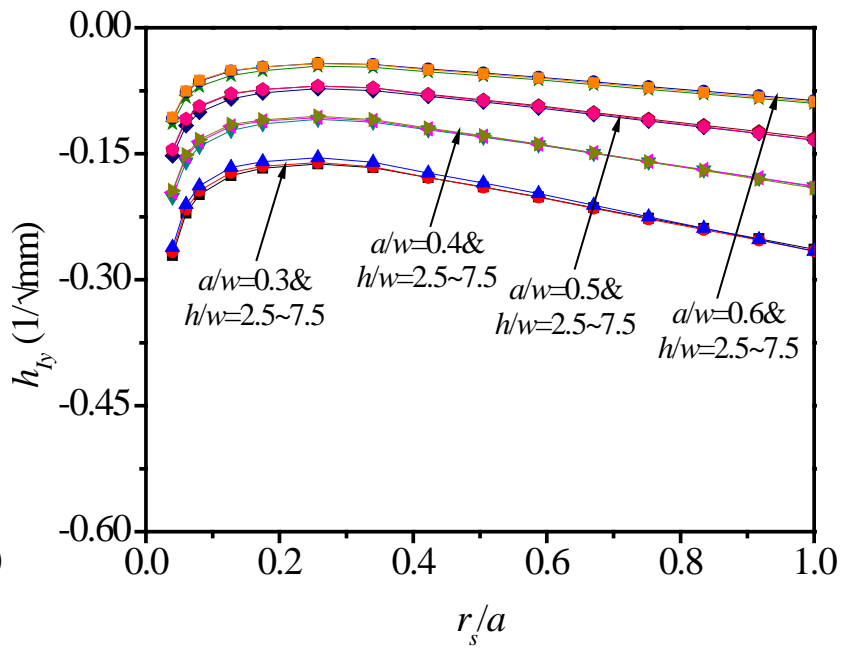

(b)

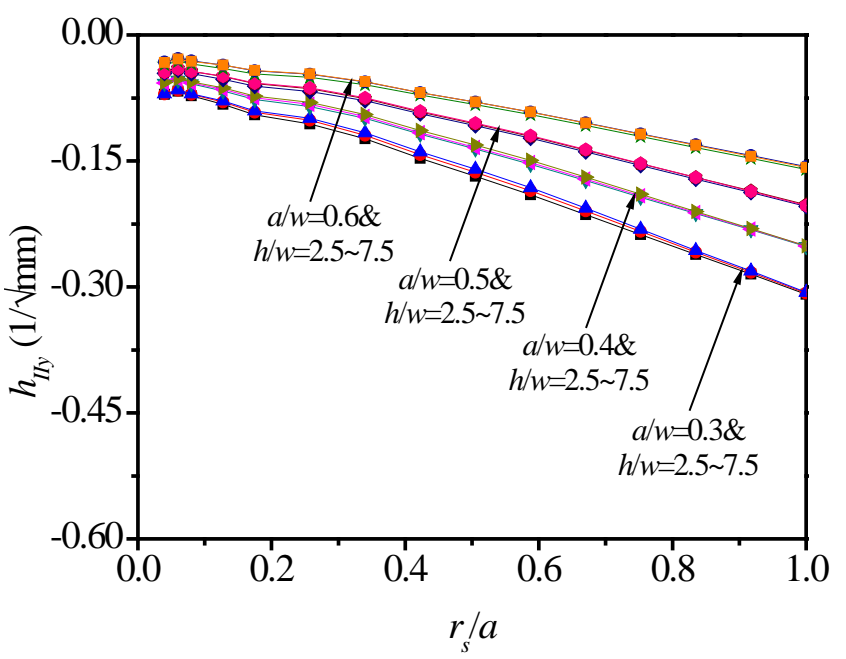

(d)

Figure 14 Nodal weight functions on lower crack-face 


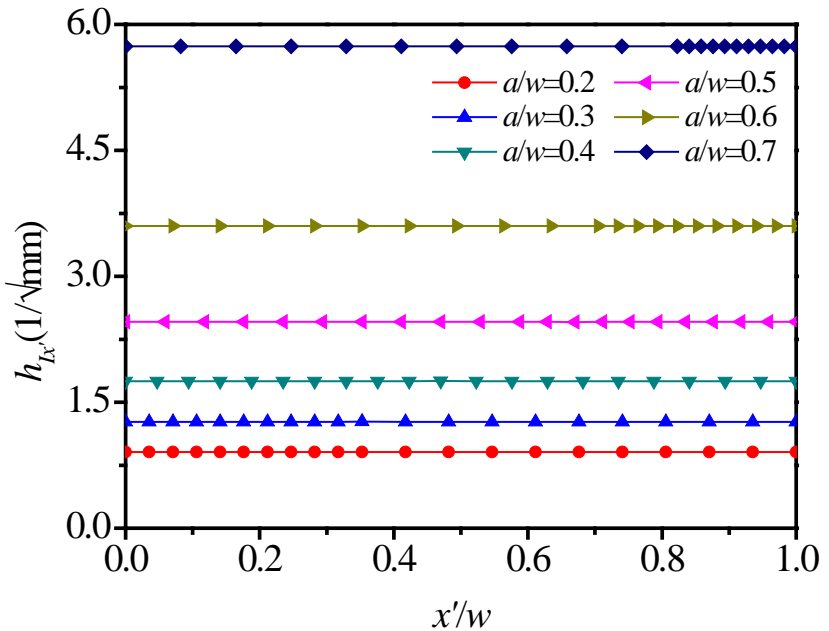

(a)

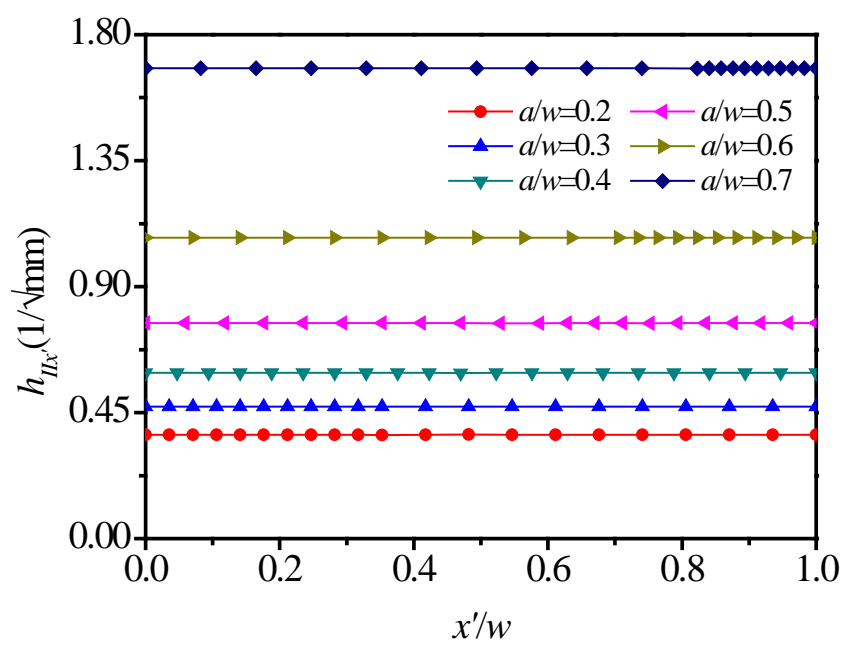

(c)

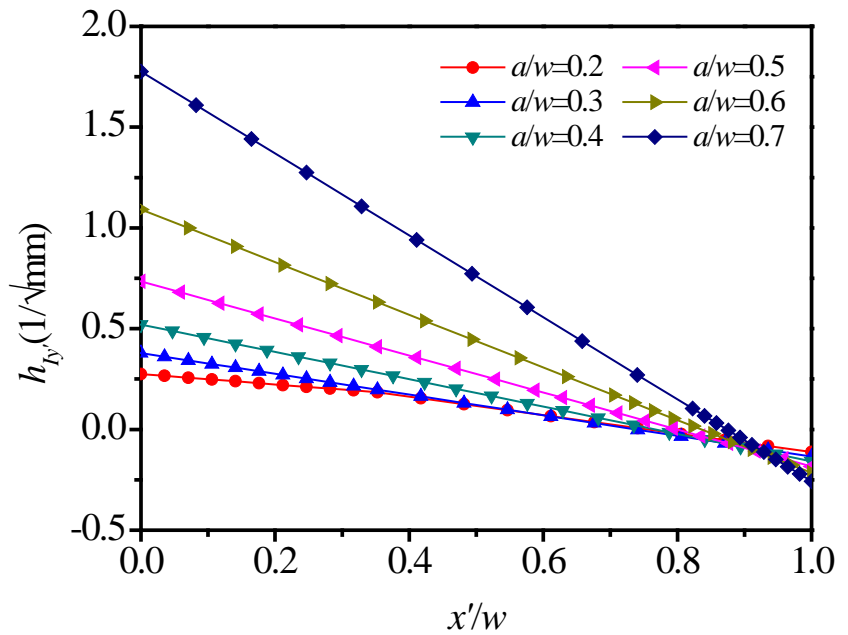

(b)

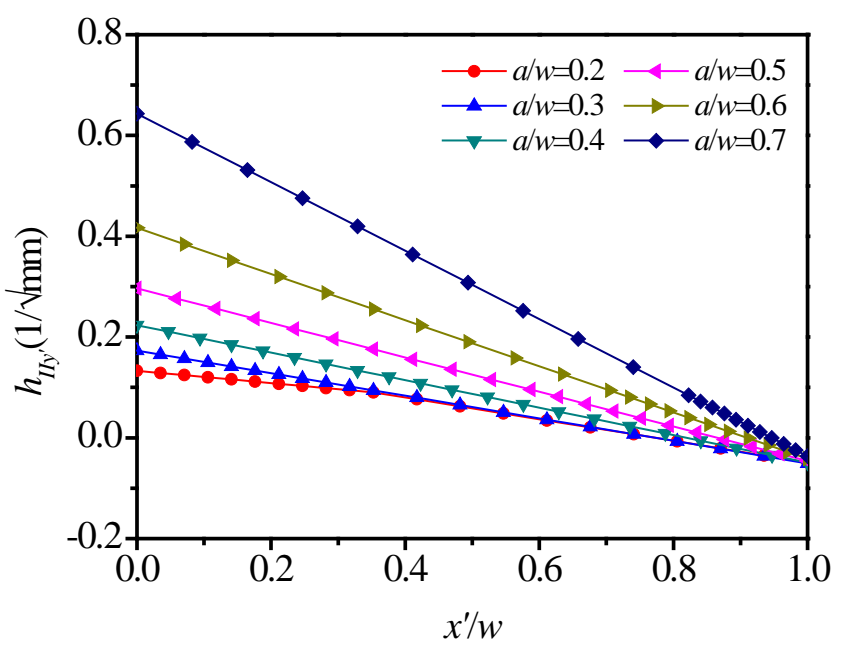

(d)

Figure 15 Nodal weight functions on top-face 


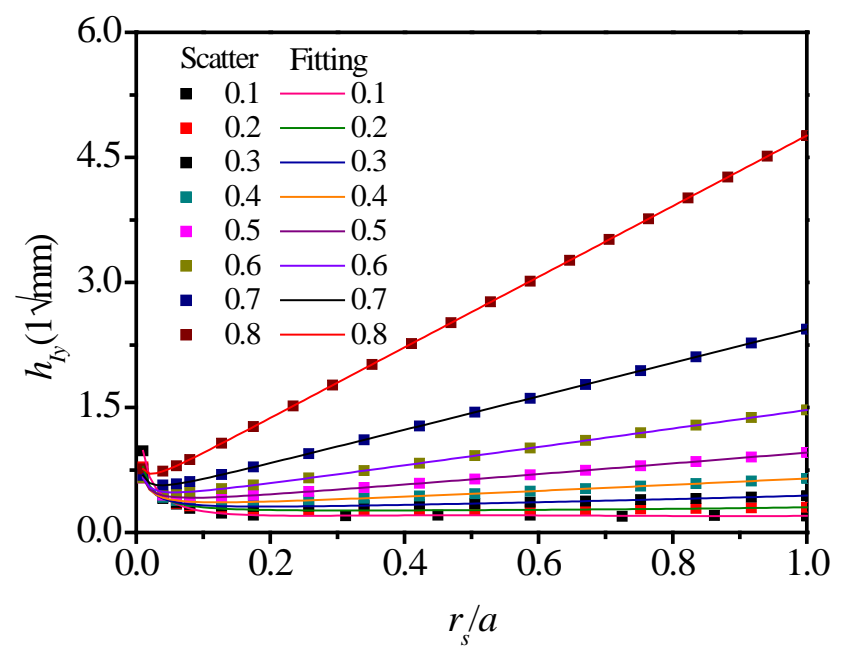

(a) $h_{i y}$ upper crack face

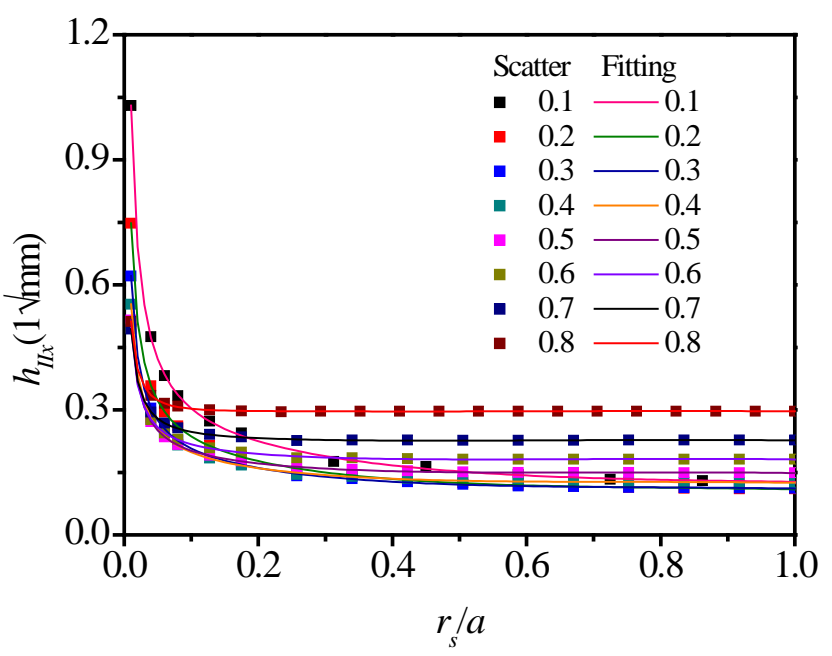

(c) $h_{i i x}$ upper crack face

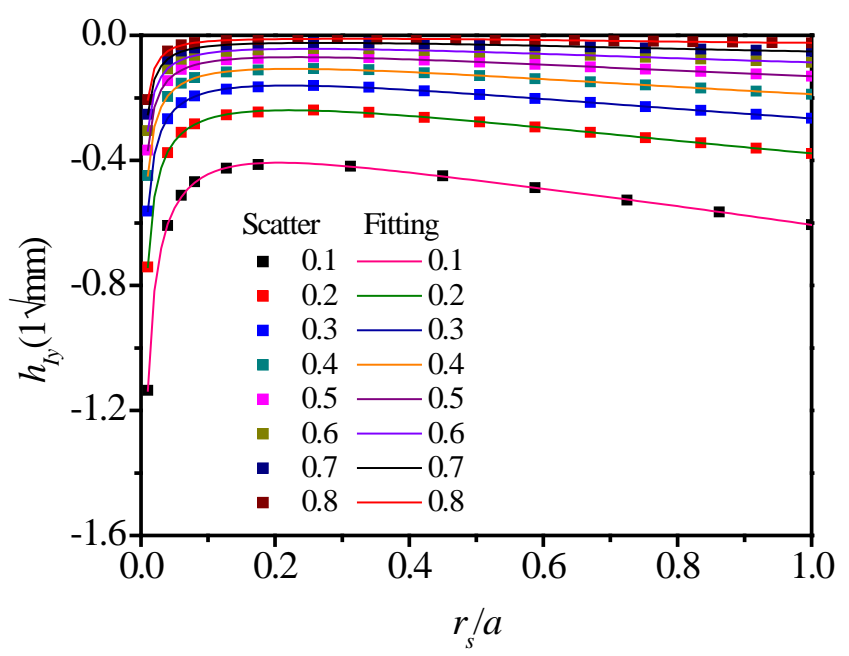

(b) $h_{i y}$ lower crack face

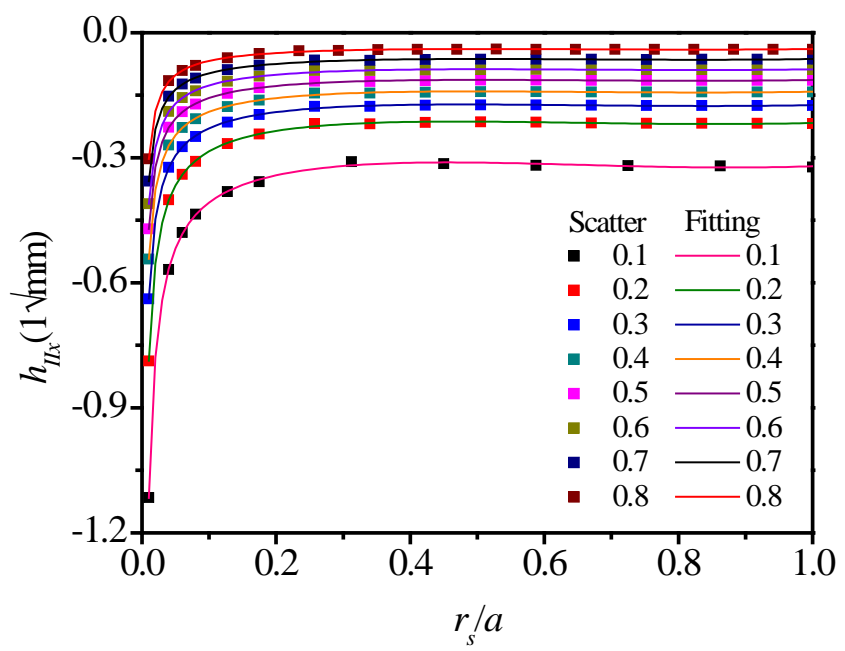

(d) $h_{i i x}$ lower crack face

Figure 16 Primary nodal weight functions and fitting primary weight functions 


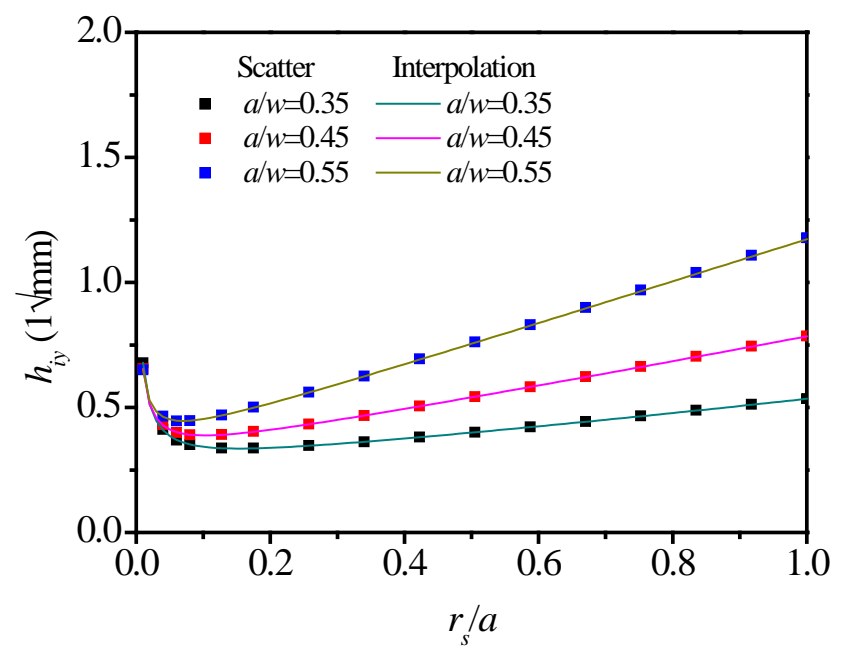

(a) $h_{i y}$ upper crack face

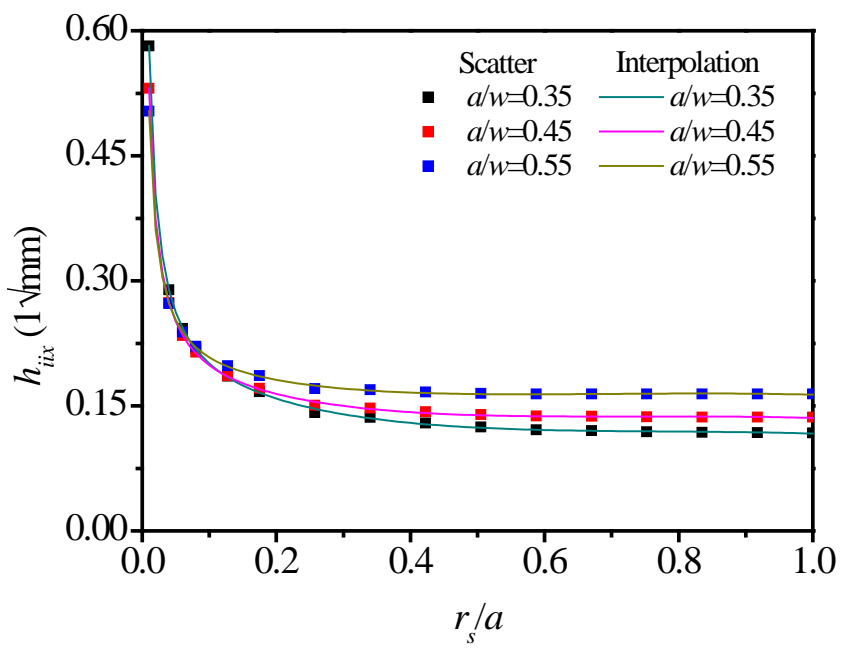

(c) $h_{i i x}$ upper crack face

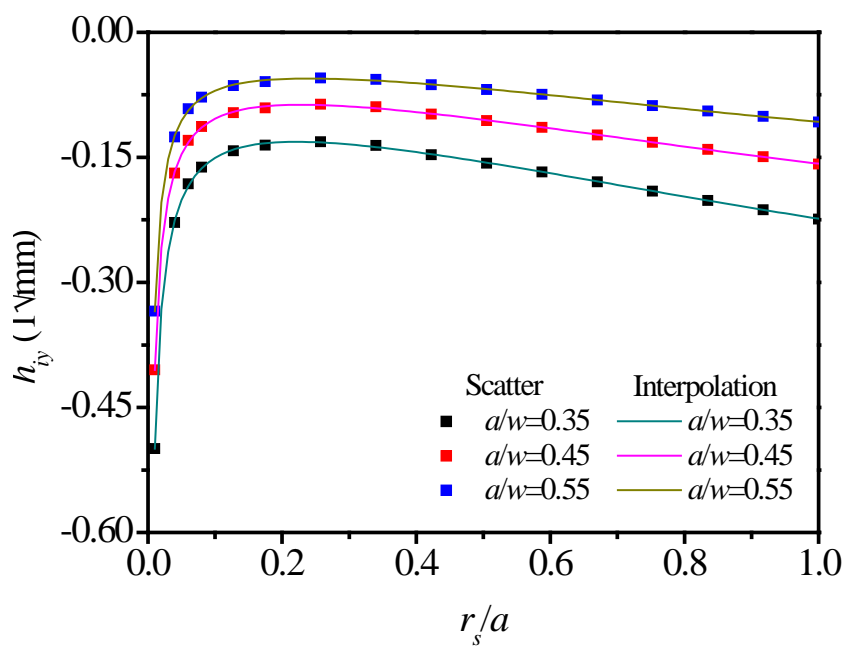

(b) $h_{i y}$ lower crack face

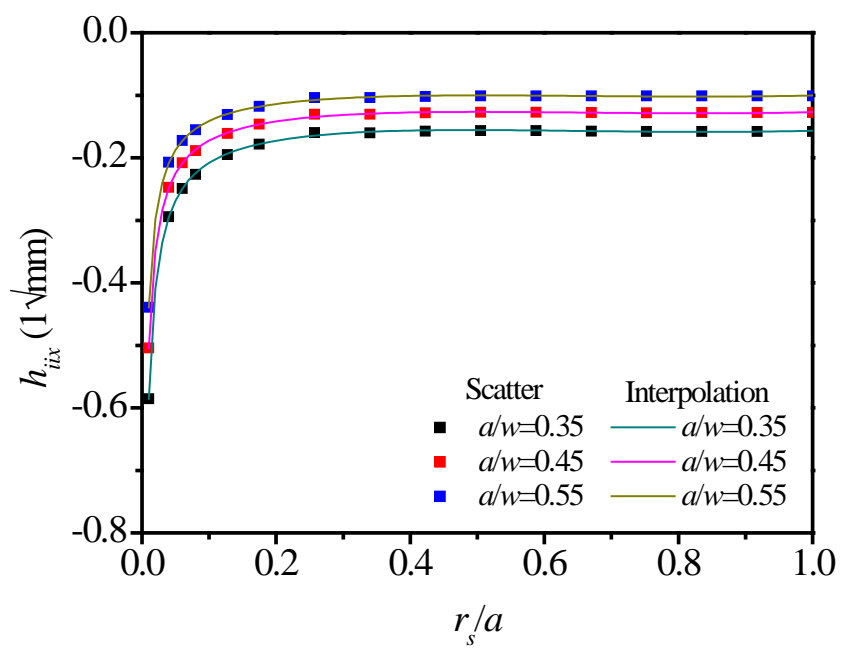

(d) $h_{i i x}$ lower crack face

Figure 17 Primary weight functions from interpolated results and the VCE technique 


\section{TABLES}

Table 1 Strain energy release rates calculated by different methods

\begin{tabular}{|c|c|c|c|c|c|}
\hline \multirow{2}{*}{$a / w$} & \multicolumn{3}{|c|}{ Virtual Crack Extension } & \multirow{2}{*}{$\mathrm{J} /\left(\sigma_{\theta}{ }^{2} a / E\right)$} & \multirow{2}{*}{$\begin{array}{c}\text { Discrepancy } \\
(\%)\end{array}$} \\
\cline { 2 - 4 } & $\mathrm{G}_{\mathrm{I}} /\left(\sigma_{\theta}{ }^{2} a / E\right)$ & $\mathrm{G}_{\mathrm{II}} /\left(\sigma_{\theta}{ }^{2} a / E\right)$ & $\mathrm{G} /\left(\sigma_{\theta}{ }^{2} a / E\right)$ & 1.579 & -0.204 \\
\hline 0.1 & 1.264 & 0.318 & 1.582 & 1.617 & -1.057 \\
\hline 0.2 & 1.339 & 0.295 & 1.635 & 1.861 & -0.432 \\
\hline 0.3 & 1.571 & 0.298 & 1.869 & 2.381 & -0.307 \\
\hline 0.4 & 2.056 & 0.332 & 2.388 & 3.420 & -0.406 \\
\hline 0.5 & 3.014 & 0.420 & 3.434 & 5.687 & -0.482 \\
\hline 0.6 & 5.090 & 0.625 & 5.714 & 11.680 & -0.483 \\
\hline 0.7 & 10.558 & 1.178 & 11.736 & 34.551 & 0.029 \\
\hline 0.8 & 31.312 & 3.229 & 34.541 & \\
\hline
\end{tabular}

Note: (1) Virtual Crack Extension: Strain energy release rates determined from applying the VCE technique into finite element;

(2) J: Strain energy release rates determined based on the J-Integral method. 
Table 2 Strain energy release rates calculated by different methods

\begin{tabular}{|c|c|c|c|c|c|}
\hline \multirow{2}{*}{$a / w$} & \multicolumn{3}{|c|}{ Weight Function method } & \multirow{2}{*}{$\mathrm{J} /\left(\sigma^{2} a / E\right)$} & \multirow{2}{*}{ Discrepancy (\%) } \\
\cline { 2 - 4 } & $\mathrm{G}_{\mathrm{I}} /\left(\sigma^{2} a / E\right)$ & $\mathrm{G}_{\mathrm{II}} /\left(\sigma^{2} a / E\right)$ & $\mathrm{G} /\left(\sigma^{2} a / E\right)$ & & \\
\hline 0.2 & 2.136 & 0.568 & 2.704 & 2.686 & -0.679 \\
\hline 0.3 & 3.187 & 0.802 & 3.990 & 3.976 & -0.352 \\
\hline 0.4 & 5.283 & 1.219 & 6.502 & 6.487 & -0.238 \\
\hline 0.5 & 9.754 & 2.022 & 11.776 & 11.746 & -0.262 \\
\hline 0.6 & 20.597 & 3.775 & 24.372 & 24.295 & -0.319 \\
\hline 0.7 & 53.019 & 8.457 & 61.476 & 61.297 & -0.293 \\
\hline
\end{tabular}

Note: (1) Weight Function method: Strain energy release rates determined based on the weight function method employing the weight function from the pure bending load;

(2) J: Strain energy release rates determined based on the J-Integral method. 
Table 3 Coefficients of Eq.(31) for crack-face weight function

(a) $h_{\text {iy }}$ on upper crack face

\begin{tabular}{|c|c|c|c|c|c|c|}
\hline$a / w$ & $\mathrm{~A} 1$ & $\mathrm{~A} 2$ & $\mathrm{~A} 3$ & $\mathrm{~A} 4$ & $\mathrm{~A} 5$ & $\mathrm{~A} 6$ \\
\hline 0.1 & -0.092734 & -0.229009 & 2.383365 & -6.921558 & 7.379385 & -2.718690 \\
\hline 0.2 & -0.080541 & 0.038203 & -0.131954 & -0.766838 & 1.203308 & -0.564631 \\
\hline 0.3 & -0.067423 & 0.013743 & -0.420176 & 0.037796 & 0.141774 & -0.147851 \\
\hline 0.4 & -0.059254 & -0.018566 & -0.559689 & 0.303690 & -0.377589 & 0.063093 \\
\hline 0.5 & -0.053520 & -0.052535 & -0.646945 & 0.270632 & -0.652596 & 0.176646 \\
\hline 0.6 & -0.049182 & -0.090396 & -0.716896 & -0.051040 & -0.796518 & 0.234919 \\
\hline 0.7 & -0.045670 & -0.139725 & -0.783600 & -0.878386 & -0.835279 & 0.244057 \\
\hline 0.8 & -0.043304 & -0.204715 & -0.958865 & -2.807243 & -1.055604 & 0.308214 \\
\hline
\end{tabular}

(b) $h_{i y}$ on lower crack face

\begin{tabular}{|c|c|c|c|c|c|c|}
\hline$a / w$ & $\mathrm{~A} 1$ & $\mathrm{~A} 2$ & $\mathrm{~A} 3$ & $\mathrm{~A} 4$ & $\mathrm{~A} 5$ & $\mathrm{~A} 6$ \\
\hline 0.1 & 0.111336 & 0.021621 & -0.12338 & 1.517708 & -1.50778 & 0.587017 \\
\hline 0.2 & 0.084595 & -0.17307 & 0.777969 & -1.06263 & 1.184471 & -0.43361 \\
\hline 0.3 & 0.069391 & -0.1949 & 0.726072 & -1.02747 & 1.086364 & -0.39387 \\
\hline 0.4 & 0.060614 & -0.22225 & 0.749106 & -1.12875 & 1.130974 & -0.40135 \\
\hline 0.5 & 0.054837 & -0.25015 & 0.810916 & -1.2877 & 1.228063 & -0.42502 \\
\hline 0.6 & 0.050751 & -0.27922 & 0.904473 & -1.49009 & 1.359472 & -0.45891 \\
\hline 0.7 & 0.04775 & -0.3123 & 1.039534 & -1.76236 & 1.552487 & -0.51361 \\
\hline 0.8 & 0.044793 & -0.34309 & 1.199906 & -2.10641 & 1.836285 & -0.60747 \\
\hline
\end{tabular}


(c) $h_{\text {iix }}$ on upper crack face

\begin{tabular}{|c|c|c|c|c|c|c|}
\hline$a / w$ & B1 & B2 & B3 & B4 & B5 & B6 \\
\hline 0.1 & 0.131802 & -0.44081 & 1.833506 & -3.32233 & 2.800416 & -0.87519 \\
\hline 0.2 & 0.091909 & -0.28242 & 1.354536 & -2.71649 & 2.529538 & -0.86776 \\
\hline 0.3 & 0.076298 & -0.24426 & 1.255316 & -2.54506 & 2.411011 & -0.84208 \\
\hline 0.4 & 0.066731 & -0.20921 & 1.163286 & -2.34709 & 2.250624 & -0.79935 \\
\hline 0.5 & 0.060091 & -0.17488 & 1.083916 & -2.13085 & 2.043538 & -0.7334 \\
\hline 0.6 & 0.055304 & -0.1429 & 1.034728 & -1.92274 & 1.798936 & -0.64223 \\
\hline 0.7 & 0.052347 & -0.12152 & 1.086784 & -1.86518 & 1.633669 & -0.55949 \\
\hline 0.8 & 0.051027 & -0.11057 & 1.317747 & -2.13715 & 1.715409 & -0.5401 \\
\hline
\end{tabular}

(d) Table $h_{i i x}$ on lower crack face

\begin{tabular}{|c|c|c|c|c|c|c|}
\hline$a / w$ & B1 & B2 & B3 & B4 & B5 & B6 \\
\hline 0.1 & -0.13498 & 0.427064 & -2.35207 & 4.672481 & -4.70868 & 1.775827 \\
\hline 0.2 & -0.09009 & 0.212744 & -1.2018 & 2.357066 & -2.45043 & 0.956048 \\
\hline 0.3 & -0.07384 & 0.182489 & -0.99382 & 1.893965 & -1.92035 & 0.737893 \\
\hline 0.4 & -0.06439 & 0.176023 & -0.90237 & 1.703452 & -1.69844 & 0.644279 \\
\hline 0.5 & -0.0581 & 0.182118 & -0.86356 & 1.629122 & -1.60587 & 0.602862 \\
\hline 0.6 & -0.05369 & 0.198288 & -0.86797 & 1.644464 & -1.60506 & 0.596248 \\
\hline 0.7 & -0.05062 & 0.22618 & -0.92195 & 1.753932 & -1.68949 & 0.618835 \\
\hline 0.8 & -0.04719 & 0.244479 & -0.90486 & 1.673915 & -1.56931 & 0.563969 \\
\hline
\end{tabular}

